NASACONTRACTOR REPOR T

$\infty$
$\vdots$
$\vdots$
$\vdots$
$\Sigma$

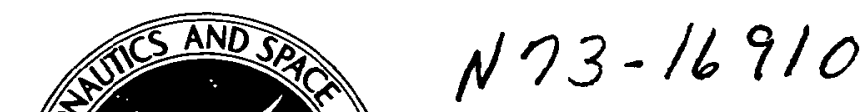
NASA CR-2181

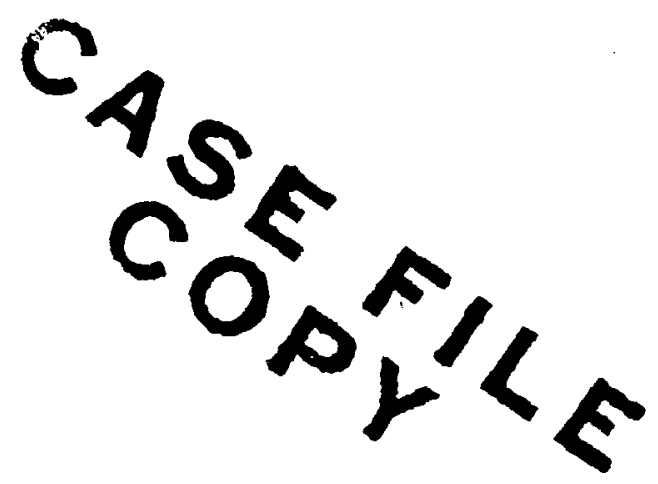

TWO BONDED HALF PLANES WITH

A CRACK GOING THROUGH THE INTERFACE

by F. Erdogan and V. Biricikoglu

Prepared by

LEHIGH UNIVERSITY

Bethlehem, Pa.

for Langley Research Center

NATIONAL AERONAUTICS AND SPACE ADMINISTRATION - WASHINGTON, D. C. - FEBRUARY 1973 


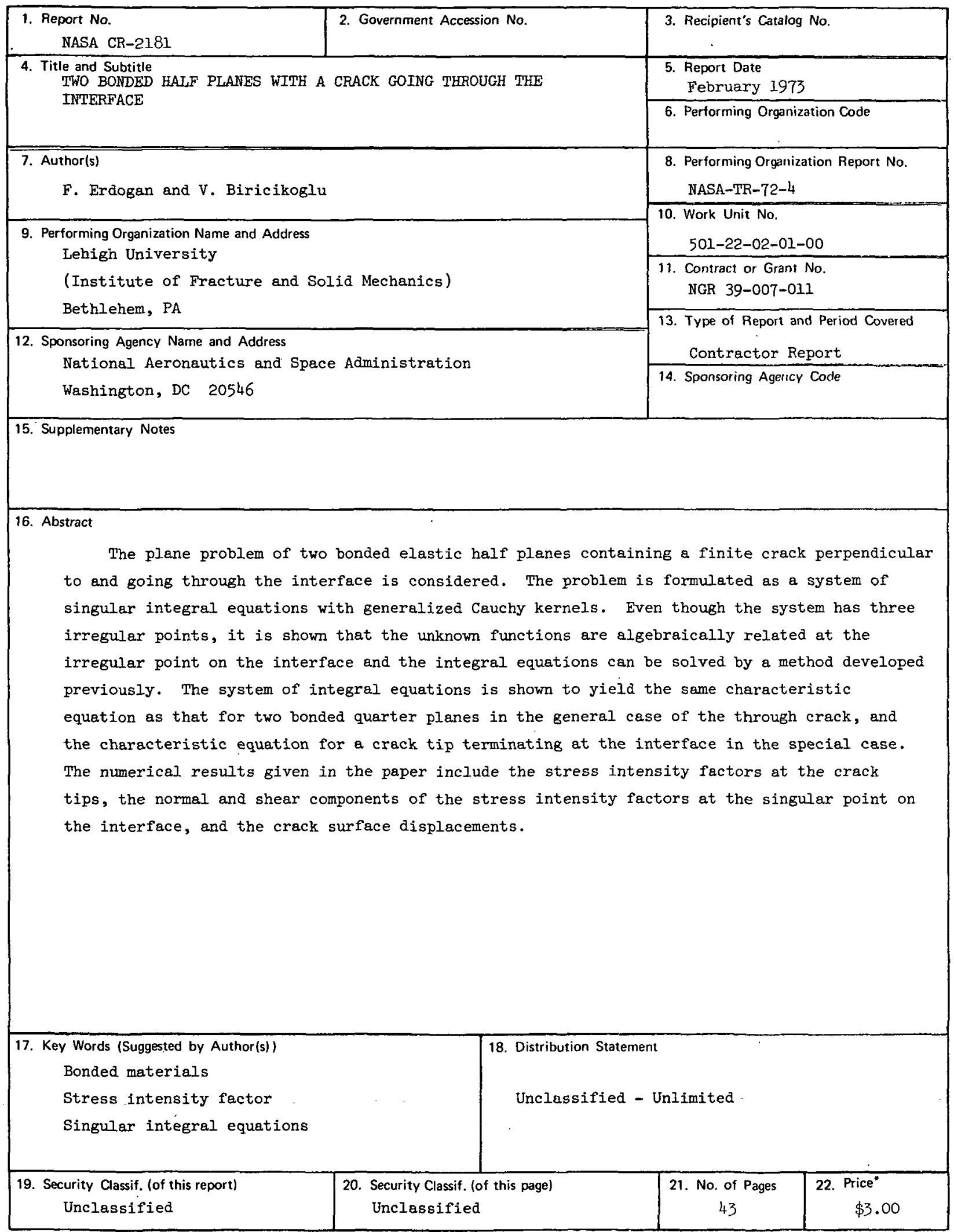

"For sale by the National Technical Information Service, Springfield, Virginia 22151 


\title{
TWO BONDED HALF PLANES WITH A \\ CRACK GOING THROUGH THE INTERFACE*
}

\author{
BY \\ F. ERDOGAN AND V. BiriciKogLU \\ Lehigh University, Bethlehem, Pennsylvania
}

\begin{abstract}
The plane problem of two bonded elastic half planes containing a finite crack perpendicular to and going through the interface is considered. The problem is formulated as a system of singular integral equations with generalized Cauchy kernels. Even though the system has three irregular points, it is shown that the unknown functions are algebraically related at the irregular point on the interface and the integral equations can be solved by a method developed previously. The system of integral equations is shown to yield the same characteristic equation as that for two bonded quarter planes in the general case of the through crack, and the characteristic equation for a crack tip terminating at the interface in the special case. The numerical results given in the paper include the stress intensity factors at the crack tips, the normal and shear components of the stress intensity factors at the singular point on the interface, and the crack surface displacements.
\end{abstract}

* This work was supported by the National Science Foundation under the Grant GK 11977, and by the National Aeronautics and Space Administration under the Grant NGR-39-007-011. 
1. INTRODUCTION

In considering the fracture of composite materials, one may approach the problem from two different points of view. In the first approach the primary interest is in studying and in estimating the "bulk strength" of the given structure under a known system of external loads and environmental conditions. In this type of studies it is usually assumed that the existing imperfections such as voids, cracks, and inclusions are randomly distributed throughout the composite medium and the material is statistically homogeneous. Thus, the very nature of the problem requires that some $k$ ind of a statistical strength theory be used as a guide in the investigations.

In the second approach to studying the fracture of composites, one is basically interested in the initiation of fracture from the "localized" imperfections which are known (or assumed) to exist in the material. In this type of studies it is usually assumed that the composite medium consists of perfectly bonded elastic components and the localized imperfection may be idealized as a plane crack or as a flat elastic inclusion. The problem of a finite crack lying parallel to or at a bimaterial interface in composites with various geometries was discussed in [1-5]. The similar problem for a flat inclusion was studied in [6]. An up-to-day review of the general fracture problems in composite materials and a summary of some of the known results may be found in [7]. The distinguishing feature of the solutions given in [1-7] as well as the other 
known solutions which appeared in literature within the past decade is that the strength of the stress singularity at the imperfection front is $-1 / 2$ and, in the case of a crack, the quasi-static stress state in the neighborhood of the crack front remains autonomous as the crack propagates. That is, aside from a slight change in a multiplicative constant known as the stress intensity factor, the asymptotic nature of the stress state in the vicinity of the crack front remains unchanged.

On the other hand, when the crack front terminates at a bi-material interface in the composite medium the strength of the stress singularity is no longer $-1 / 2$ and the angular distribution of stresses differs considerabiy from that of a crack tip imbedded in a homogeneous medium. After reaching the interface, further propagation of the crack may be in the form of (a) a cleavage crack into the second medium (Figure 1), (b) a debonding crack along the interface, or (c) a "reflected" crack back into the first medium. The basic problem of a finite crack terminating at a bi-material interface was discussed in some detail in a previous paper [8]. [8] also presents a tentative fracture criterion which may be used in predicting the mode of the fracture propagation (such as $(a)-(c)$, mentioned above) as well as the level of the external loads initiating the fracture provided the fracture strengths of the two adjoining materials and of the interface are known.

In this paper we consider the problem of a crack going 
through the interface into the second medium. From the analytical viewpoint the unusual feature of the problem is that it has three irregular points (two crack tips and the intersection of the crack with the interface) at which the stress state is generally singular. One of the objectives of the paper is the development of the necessary theoretical and numerical techniques for handling problems of this nature.

\section{THE INTEGRAL EQUATIONS}

Consider the plane elasticity problem shown in figure 1. In this paper only the symmetric problem will be considered; that is, it is assumed that in the composite medium without the crack and subjected to the same external loads as the cracked medium, the planes $(r>0, \theta=\pi)$ and $(r>0, \theta=0)$ are free from shear and are acted upon by normal stresses $p_{1}(r)$ and $p_{2}(r)$, respectively. Thus, through a proper superposition, the singular part of the problem may be reduced to that shown in Figure 1, where the self-equilibrating crack surface tractions $-p_{1}(r)$ and $-p_{2}(r)$ are the only external loads. In the special case of uniform loading $p_{1}$ and $p_{2}$ are constant and the continuity conditions require that

$$
\begin{aligned}
& \frac{p_{1}}{E_{1}}=\frac{p_{2}}{E_{2}} \text {, for } p l a n e \text { stress, } \\
& \frac{1-v_{1}^{2}}{E_{1}} p_{1}=\frac{1-v_{2}^{2}}{E_{2}} p_{2} \text {, for } p l a n e \text { strain. }
\end{aligned}
$$

To solve the problem shown in Figure 1 we will first 


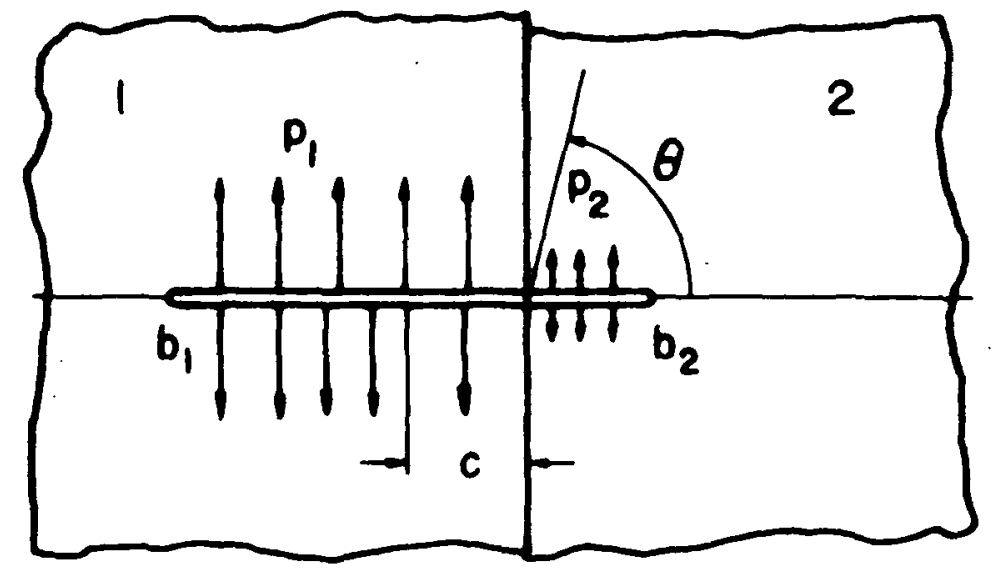

Figure 1. The geometry of the crack going through the
interface of two bonded elastic half planes.

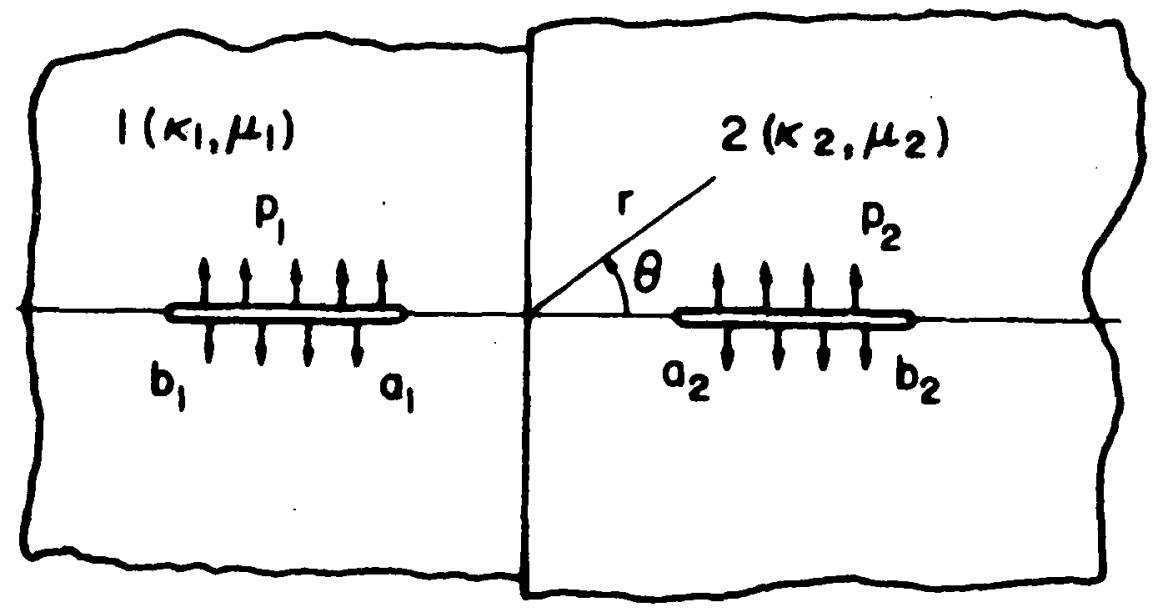

Figure 2. Two cracks imbedded into adjacent half planes. 
consider the problem described in Figure 2 where each half plane contains a finite crack perpendicular to the interface, and then let the distances $a_{1}$ and $a_{2}$ go to zero.

Because of symmetry, it is sufficient to consider the problem in the half plane $0 \leq r<\infty, 0<\theta<\pi$ (Figure 2). The related elasticity problem has to be solved under the following boundary conditions:

$$
\begin{aligned}
& \tau_{1 r \theta}(r, \pi)=0, \quad 0 \leq r<\infty, \\
& \tau_{1 \theta \theta}(r, \pi-0)=-p_{1}(r), \quad a_{1}<r<b_{1}, \\
& u_{1 \theta}(r, \pi)=0, \quad 0 \leq r<a_{1}, b_{1}<r<\infty ; \\
& u_{1 \theta}=u_{2 \theta}, \quad u_{1 r}=u_{2 r}, \quad \tau_{1 \theta \theta}=\tau_{2 \theta \theta}, \\
& \tau_{1 r \theta}=\tau_{2 r \theta}, \quad \theta=\pi / 2, \quad 0 \leq r<\infty ; \\
& \tau_{2 r \theta}(r, 0)=0, \quad 0 \leq r<\infty, \\
& \tau_{2 \theta \theta}(r,+0)=-p_{2}(r), \quad a_{2}<r<b_{2}, \\
& u_{2 \theta}(r ; 0)=0, \quad 0 \leq r<a_{2}, \quad b_{2}<r<\infty .
\end{aligned}
$$

Let the unknown functions $f_{1}$ and $f_{2}$ be defined as follows:

$$
\begin{aligned}
& f_{1}(r)=-2 \frac{\partial}{\partial r} u_{1 \theta}(r, \pi-0), \\
& f_{2}(r)=2 \frac{\partial}{\partial r} u_{2 \theta}(r,+0) .
\end{aligned}
$$

From (2.c), (4.c) and (5) it follows that 


$$
\begin{array}{ll}
f_{1}(r)=0, & 0 \leq r<a_{1}, \quad b_{1}<r<\infty, \\
f_{2}(r)=0, & 0 \leq r<a_{2}, \quad b_{2}<r<\infty, \\
\int_{a_{1}}^{b_{1}} f_{1}(r) d r=0, & \int_{a_{2}}^{b_{2}} f_{2}(r) d r=0 .
\end{array}
$$

Using Mellin transforms and following the procedure outlined in [8], after somewhat routine manipulations the problem can be reduced to the following system of integral equations for the functions $f_{1}$ and $f_{2}$ :

$$
\begin{aligned}
& \frac{1}{\pi} \int_{a_{1}}^{b_{1}} \frac{f_{1}(s)}{s-r} d s+\frac{1}{\pi} \int_{a_{1}}^{b_{1}} k_{11}(r, s) f_{1}(s) d s \\
& \quad+\frac{1}{\pi} \int_{a_{2}}^{b_{2}} k_{12}(r, s) f_{2}(s) d s=-\frac{1+k_{1}}{2 \mu 1} p_{1}(r), \quad a_{1}<r<b_{1}, \\
& \frac{1}{\pi} \int_{a_{2}}^{b_{2}} \frac{f_{2}(s)}{s-r} d s+\frac{1}{\pi} \int_{a_{1}}^{b_{1}} k_{21}(r, s) f_{1}(s) d s \\
& \quad+\frac{1}{\pi} \int_{a_{2}}^{b_{2}} k_{22}(r, s) f_{2}(s) d s=-\frac{1+k_{2}}{2 \mu_{2}} p_{2}(r), \quad a_{2}<r<b_{2},
\end{aligned}
$$

where the kernels $k_{i j}$ are given by

$$
\begin{aligned}
& k_{11}(r, s)=\sum_{k=1}^{3} \frac{c_{1 k^{r}} r^{k-1}}{(s+r)^{k}}, \quad k_{22}(r, s)=\sum_{k=1}^{3} \frac{c_{2 k} r^{k-1}}{(s+r)^{k}}, \\
& k_{12}(r, s)=\sum_{1}^{2} \frac{d_{1 k} r^{k-1}}{(s+r)^{k}}, \quad k_{21}(r, s)=\sum_{1}^{2} \frac{d_{2 k} r^{k-1}}{(s+r)^{k}} ;
\end{aligned}
$$




$$
\begin{aligned}
& c_{11}=\frac{1}{2}-\frac{m_{1}\left(1+k_{1}\right)}{2\left(m_{1}+k_{2}\right)}-\frac{3\left(1-m_{1}\right)}{2\left(1+m_{1} k_{1}\right)} \text {. } \\
& c_{12}=\frac{6\left(1-m_{1}\right)}{1+m_{1} k_{1}}, \quad c_{13}=-\frac{4\left(1-m_{1}\right)}{1+m_{1} k_{1}} \text {; } \\
& c_{21}=\frac{1}{2}-\frac{m_{2}\left(1+\kappa_{2}\right)}{2\left(m_{2}+\kappa_{1}\right)}-\frac{3\left(1-m_{2}\right)}{2\left(1+m_{2} k_{2}\right)} \text {, } \\
& c_{22}=\frac{6\left(1-m_{2}\right)}{1+m_{2} k_{2}}, \quad c_{23}=-\frac{4\left(1-m_{2}\right)}{1+m_{2} k_{2}} ; \\
& d_{11}=\frac{3\left(1+k_{1}\right)}{2\left(m_{2}+k_{1}\right)}-\frac{1+k_{1}}{2\left(1+m_{2} k_{2}\right)}, \quad d_{12}=\frac{1+k_{1}}{1+m_{2} k_{2}}-\frac{1+k_{1}}{m_{2}+k_{1}} ; \\
& d_{21}=\frac{3\left(1+\kappa_{2}\right)}{2\left(m_{1}+k_{2}\right)}-\frac{1+\kappa_{2}}{2\left(1+m_{1} \kappa_{1}\right)}, \quad d_{22}=\frac{1+k_{2}}{1+m_{1} \kappa_{1}}-\frac{1+\kappa_{2}}{m_{1}+k_{2}} ; \\
& m_{1}=\mu_{2} / \mu_{1}, \quad m_{2}=\mu_{1} / \mu_{2} \text {. }
\end{aligned}
$$

In (9) $\mu_{1}$ and $\mu_{2}$ are the shear moduli, $\kappa_{i}=3-4 \nu_{i}$ for plane strain and $\kappa_{i}=\left(3-v_{i}\right) /\left(1+v_{i}\right)$ for the generalized plane stress, $(i=1,2), v_{i}$ being the Poisson's ratio.

From (8) it is seen that for $a_{1}>0, a_{2}>0$ the kernels $k_{i j}(r, s),(i, j=1,2)$ are bounded and continuous. Hence (7) is a simple system of singular integral equations with an index $\kappa=1$ and the additional conditions (6.c and $d$ ) which are required for a unique solution. These equations can be solved for the unknown functions $f_{1}$ and $f_{2}$ in a standard manner. A simple and highly effective numerical method has been described in [9]. After obtaining $f_{1}$ and $f_{2}$, all the desired field quantities may be expressed as and evaluated from the definite 
integrals with kernels corresponding to the particular field quantity and the density functions $f_{i},(i=1,2)$ (see, for example, [8] for the case of one crack). From the practical viewpoint of particular interest are the stress intensity factors defined by ${ }^{*}$

$$
\begin{aligned}
& k\left(a_{1}\right)=\lim _{r \rightarrow a_{1}} \sqrt{2\left(a_{1}-r\right)} \tau_{1 \theta \theta}(r, \pi)=\lim _{r \rightarrow a_{1}} \frac{2 \mu_{1}}{\sqrt{+k_{1}}} \sqrt{2\left(r-a_{1}\right) f_{1}}(r), \\
& k\left(b_{1}\right)=\lim _{r \rightarrow b_{1}} \sqrt{2\left(r-b_{1}\right)} \tau_{1 \theta \theta}(r, \pi)=-\lim _{r \rightarrow b_{1}} \frac{2 \mu_{1}}{\sqrt{+k_{1}}} \sqrt{2\left(b_{1}-r\right)} f_{1}(r), \\
& k\left(a_{2}\right)=\lim _{r \rightarrow a_{2}} \sqrt{2\left(a_{2}-r\right)} \tau_{2 \theta \theta}(r, 0)=\lim _{r \rightarrow a_{2}} \frac{2 \mu_{2}}{\sqrt{+k_{2}}} \sqrt{2\left(r-a_{2}\right)} f_{2}(r), \\
& k\left(b_{2}\right)=\lim _{r \rightarrow b_{2}} \sqrt{2\left(r-b_{2}\right)} \tau_{2 \theta \theta}(r, 0)=-\lim _{r \rightarrow b_{2}} \frac{2 \mu_{2}}{\sqrt{+k_{2}}} \sqrt{2\left(b_{2}-r\right)} f_{2}(r) .
\end{aligned}
$$

For the bonded planes Material 1 Aluminum $\left(E_{1}=10^{7}\right.$ psi, $\left.v_{1}=0.3\right)$, and Material 2 Epoxy $\left(E_{2}=4.45 \times 10^{5}\right.$ psi, $\left.\nu_{2}=0.35\right)$, Tables 1 and 2 show some of the calculated plane strain results. In these tables $p_{1}$ and $p_{2}$ refer to constant pressures applied to the crack surfaces and are related by (1). As a basis of comparison the special case of a homogeneous plane containing two collinear cracks may be mentioned. For $\mu_{1}=\mu_{2}, \nu_{1}=v_{2}$, $a_{1}=a_{2}=a, b_{1}=b_{2}=b$, the stress intensity factors are given by

\footnotetext{
¿ See Section 4 below.
} 
Table 1. Stress Intensity Factors for $a_{1}>0, a_{2}>0$. Materia 1-1:-A7uminum,-Materi-a--2:- E-poxy;

$a_{2}=1, \ell_{2}=\left(b_{2}-a_{2}\right) / 2=1 / 2$,

$c_{1}=\left(b_{1}+a_{1}\right) / 2=1.5, \quad l_{1}=\left(b_{1}-a_{1}\right) / 2$ variable.

\begin{tabular}{|c|c|c|c|c|}
\hline$l_{1}$ & $\frac{k\left(a_{1}\right)}{p_{1} \sqrt{l_{1}}}$ & $\frac{k\left(b_{1}\right)}{p_{1} \sqrt{l_{1}}}$ & $\frac{k\left(a_{2}\right)}{p_{2} \sqrt{l_{2}}}$ & $\frac{k}{p_{2} \sqrt{l_{2}}}$ \\
\hline 0.05 & 1.0015 & 1.0014 & 0.97417 & 0.98165 \\
0.10 & 1.0023 & 1.0021 & 0.97515 & 0.98241 \\
0.15 & 1.0037 & 1.0033 & 0.97679 & 0.98369 \\
0.25 & 1.0082 & 1.0069 & 0.98212 & 0.98775 \\
0.50 & 1.0326 & 1.0231 & 1.0086 & 1.0079 \\
0.75 & 1.0836 & 1.0492 & 1.0590 & 1.0454 \\
1.00 & 1.1832 & 1.0869 & 1.1474 & 1.1089 \\
1.25 & 1.4094 & 1.1437 & 1.3118 & 1.2210 \\
1.40 & 1.8046 & 1.2012 & 1.5047 & 1.3451 \\
\hline
\end{tabular}

Table 2. Stress Intensity Factors for $a_{1}>0, a_{2}>0$. Material 1: Aluminum, Material 2: Epoxy; $a_{2}=1, \quad l_{1}=l_{2}=0.5, \quad c_{1}=\left(b_{1}+a_{1}\right) / 2$ variable.

\begin{tabular}{|c|c|c|c|c|}
\hline$c_{1}$ & $\frac{k\left(a_{1}\right)}{p_{1} \sqrt{l_{1}}}$ & $\frac{k\left(b_{1}\right)}{p_{1} \sqrt{l_{1}}}$ & $\frac{k\left(a_{2}\right)}{p_{2} \sqrt{l_{2}}}$ & $\frac{k\left(b_{2}\right)}{p_{2} \sqrt{l_{2}}}$ \\
\hline 1.50 & 1.0326 & 1.0231 & 1.0086 & 1.0079 \\
1.25 & 1.0494 & 1.0326 & 1.0187 & 1.0143 \\
1.00 & 1.0844 & 1.0499 & 1.0344 & 1.0236 \\
0.75 & 1.1849 & 1.0881 & 1.0624 & 1.0393 \\
0.60 & 1.4174 & 1.1454 & 1.0965 & 1.0576 \\
\hline
\end{tabular}




$$
\begin{aligned}
& \frac{k(a)}{p_{0} \sqrt{l}}=\frac{\left[b^{2} E(m) / K(m)-a^{2}\right] \sqrt{2}}{(b-a) \sqrt{a(b+a)},} \\
&-\frac{k(b)}{p_{0} \sqrt{l}}=\frac{\sqrt{2} b^{2}[1-E(m) / K(m)]}{(b-a) \sqrt{b(b+a)}}, \\
&\left(l=(b-a) / 2, \quad m=1-a^{2} / b^{2}\right),
\end{aligned}
$$

where $p_{0}$ is the constant pressure applied to the crack surfaces and $K$ and $E$ are the complete elliptic integrals of first and second kind, respectively. For example, for $b=2 a$ which corresponds to the row $\ell_{1}=0.5$ in Table 1 (or to $c_{1}=1.5$ in Table 2), we have $k(a)=1.01762 \mathrm{p}_{0} \sqrt{l}, k(b)=1.01249 \mathrm{p}_{0} \sqrt{l}$.

As $\ell_{1} \rightarrow 1.5$ in Table 1 and $c_{1} \rightarrow 0.5$ in Table $2, k\left(a_{1}\right) \rightarrow \infty$ while the remaining three stress intensity factors approach some finite values. The reason for this is that in this limiting case $a_{1}=0$ and the singular behavior of the stresses around the crack tip is of the form $R^{-\alpha}$ with $0.5<\alpha<1$ whereas the definition of $k\left(a_{1}\right)$ is based on $R^{-0.5}$ type singularity (see (10)) where $R$ is a small distance from the crack tip $a_{1}$ (see [8]). The results for another special case are shown in Figure 3 where $a_{2}=b_{2}$. This is the problem considered in [8] where only one of the adjoining materials contains a crack. Because of the change in the power $\alpha$ of the stress singularity when the crack terminates at the interface, again as a $\rightarrow 0 k(a) \rightarrow \infty$ for $\mu_{1}>\mu_{2}$ and $k(a)+0$ for $\mu_{1}<\mu_{2}$, whereas $k(b)$ in both cases remains bounded. 


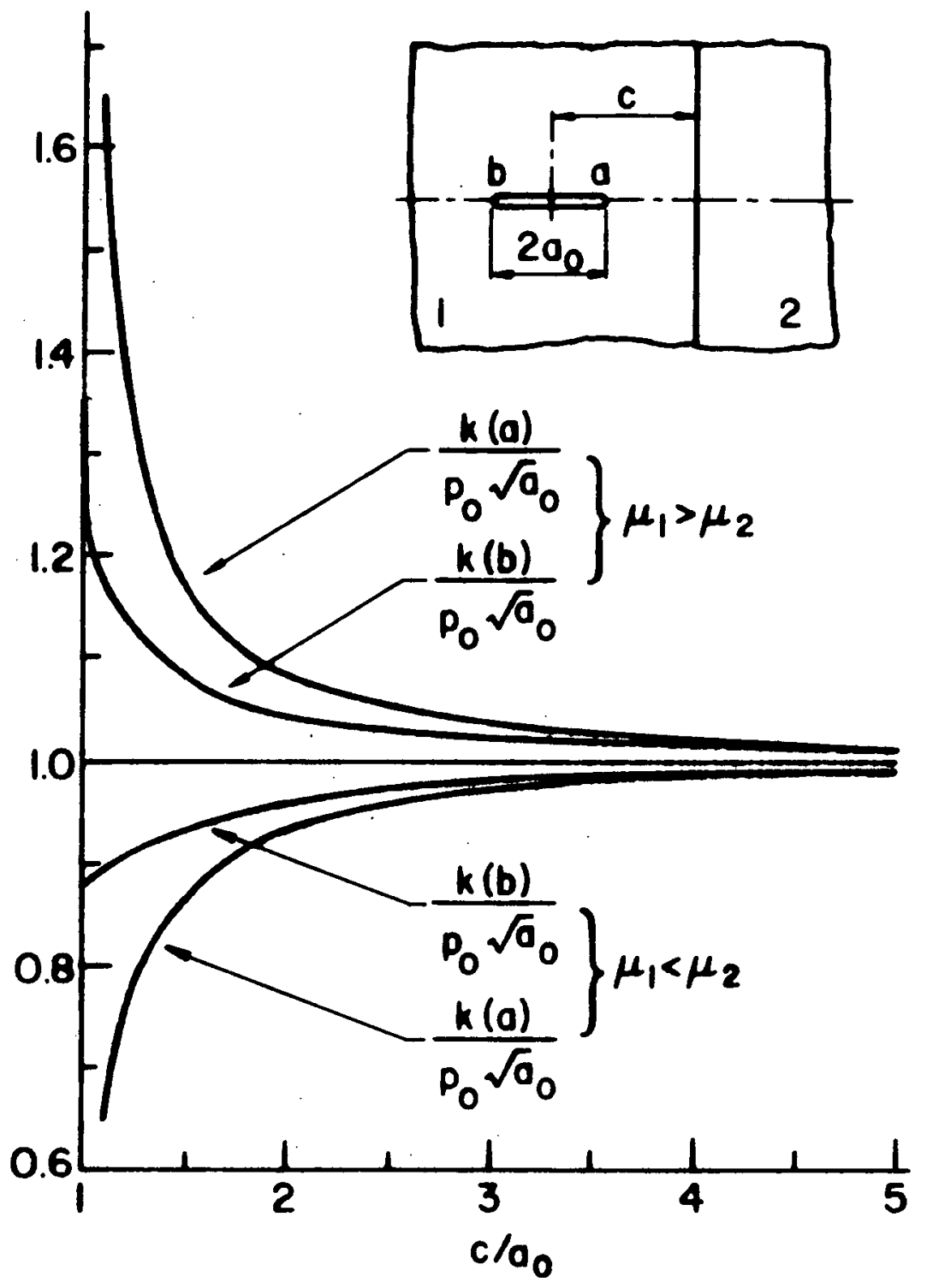

Figure 3. Stress intensity factors for a crack perpendicular to the interface (materials: aluminum and epoxy; external load: crack surface pressure $p_{0}$ ). 


\section{CRACK GOING THROUGH THE INTERFACE, $a_{1}=0=a_{2}$}

We now consider the main problem in which the crack goes through the interface, occupying both materials. In this case it is clear that the integral equations (7) are still valid, with $a_{1}=0=a_{2}$. Thus, the equations have only three irregular points $\left(b_{1}, \pi ; 0 ; b_{2}, 0\right)$, meaning that one has to be more careful in examining the singular behavior of the solution. Unlike the problem considered in the previous section for which $a_{1}>0$, $a_{2}>0$, as seen from $(8)$, here the kernels $k_{i j}$ are no longer bounded for all values of $r$ and $s$ in the closed intervals $\left[0, b_{1}\right]$ and $\left[0, b_{2}\right]$. At the irregular point 0 they become unbounded, indicating that at $r=0$ the behavior of $f_{i}(r),(i=1,2)$, will be affected by $k_{i j}(r, s)$.

To examine the behavior of $f_{1}$ and $f_{2}$ around the irregular points, following [10] we assume that the unknown functions may be expressed as

$$
\begin{aligned}
& f_{j}(s)=\frac{g_{j}(s)}{s^{\alpha}\left(b_{j}-s\right)^{\beta} j}=\frac{g_{j}(s) e^{\pi i \beta_{j}}}{s^{\alpha}\left(s-b_{j}\right)^{\beta}}, \\
& 0<\operatorname{Re}(\alpha)<1, \quad 0<\operatorname{Re}\left(\beta_{j}\right)<1, \quad(j=1,2),
\end{aligned}
$$

where $\alpha, \beta_{1}$ and $\beta_{2}$ are the powers of the singularity at the three irregular points, the function $g_{j}$ satisfies a Hölder condition in the closed interval $0 \leq s \leq b_{j},(j=1,2)$, and the function $s^{\alpha}\left(s-b_{j}\right)^{\beta} j$ is any definite branch which varies continuousiy on $0<s<b_{j},(j=1,2)$. Consider now the following sectionally holomorphic functions: 


$$
G_{j}(z)=\frac{1}{\pi-} \int_{0}^{b_{j} f_{j}(s)} \frac{1}{s-z} d s=\frac{1}{\pi} \int_{0}^{b_{j}} \frac{g_{j}(s) e^{\pi i \beta} j_{d s}}{s^{\alpha}\left(s-b_{j}\right)^{\beta}(s-z)}, \quad(j=1,2),
$$

where the complex variable $z=r+i \rho$ is defined in such a way that in each case the cut $\left(\rho=0,0<r<b_{j}\right),(j=1,2)$ lies along the positive real axis. Separating the leading terms at the end points of the cuts, (13) may be expressed as [10]

$$
G_{j}(z)=\frac{g_{j}(0) e^{\pi i \alpha}}{b_{j}{ }_{j} \operatorname{sin\pi \alpha }} \frac{l}{z^{\alpha}}-\frac{g_{j}\left(b_{j}\right)}{b_{j}{ }^{\alpha} \sin \pi \beta_{j}} \frac{1}{\left(z-b_{j}\right)^{\beta_{j}}}+G_{0 j}(z),
$$

where $G_{o j}$ is bounded everywhere except possib?y at the ends, $e_{j k}$, near which

$$
\begin{aligned}
& \left|G_{o j}(z)\right|<\frac{C_{j k}}{\left|z-e_{j k}\right| p_{j k}}, \quad(j, k=1,2) \\
& e_{j 1}=0, \quad p_{j 1}<\operatorname{Re}(\alpha) ; \quad e_{j 2}=b_{j}, \quad p_{j 2}<\operatorname{Re}\left(\beta_{j}\right),
\end{aligned}
$$

$c_{j k}$ and $p_{j k}$ being real constants.

Using now the Plemelj formula [10]

$$
\frac{1}{\pi} \int_{0}^{b} \frac{f_{j}(s)}{s-r} d s=\frac{1}{2}\left[G_{j}{ }^{+}(r)+G_{j}{ }^{-}(r)\right], \quad\left(0<r<b_{j}\right)
$$

the terms with the Cauchy singularity in (7) may be expressed as 


$$
\begin{gathered}
\frac{1}{\pi} \int_{0}^{b} \frac{f_{j}(s)}{s-r} d s=\frac{g_{j}(0)}{b_{j} \beta_{j}} \cot \pi \alpha \frac{1}{r^{\alpha}}-\frac{g_{j}\left(b_{j}\right)}{b_{j}{ }^{\alpha}} \cot \pi \beta_{j} \frac{1}{\left(b_{j}-r\right)^{\beta_{j}}} \\
\quad+G_{1 j}(r), \quad\left(0<r<b_{j}, \quad j=1,2\right),
\end{gathered}
$$

where the behavior of $G_{i j}(r)$ in the closed interval $\left[0, b_{j}\right]$ is similar to that of $G_{0 j}(j=1,2)$. The remaining terms in the integral equations (7) may be obtained directly from (14) through differentiation. From (7), (8) and (13) it is seen that in these terms $z=-r$ which is outside the cut $0<r<b_{j}$, $j=1,2$. As $r$ varies in the interval $\left(0, b_{j}\right), z$ will vary in the interval $\left(-b_{j}, 0\right)$ in which $G_{j}(z)$ is holomorphic, $(j=1,2)$. Thus, from (13) and (14) we obtain

$$
\begin{aligned}
& \frac{1}{\pi} \int_{0}^{b} \frac{f_{j}(s) d s}{s+r}=G_{j}(-r)=\frac{g_{j}(0)}{b_{j} j_{s i n \pi \alpha}} \frac{1}{r^{\alpha}}+G_{2 j}(r), \\
& \frac{1}{\pi} \int_{0}^{b} \frac{r f_{j}(s) d s}{(s+r)^{2}}=-r \frac{d}{d r} G_{j}(-r)=\frac{\alpha g_{j}(0)}{b_{j}{ }_{j} \operatorname{sin\pi \alpha }} \frac{1}{r^{\alpha}}+G_{3 j}(r), \\
& \frac{1}{\pi} \int_{0}^{b} \frac{r^{2} f_{j}(s) d s}{(s+r)^{3}}=\frac{r^{2}}{2} \frac{d^{2}}{d r^{2}} G_{j}(-r)=\frac{\alpha(\alpha+1) g_{j}(0)}{2 b_{j}{ }_{j i n \pi \alpha}} \frac{1}{r^{\alpha}}+G_{4 j}(r), \\
& \quad\left(0<r<b_{j}, j=1,2\right),
\end{aligned}
$$

where it may easily be shown that the behavior of $G_{k j}(r)$ in the closed interval $\left[0, b_{j}\right],(j=1,2, k=2,3,4)$, will again be similar to that of $G_{0 j}$ given by (15).

If we now substitute from (17), (18) and (8) into (7), 
and multiply first both sides of each equation by $r^{\alpha}$ and let $r \rightarrow 0$, then $(7 . a)$ by $\left(b_{1}-r\right)^{\beta}$ and 1 et $r \rightarrow b_{1}$, and $(7 . b)$ by $\left(b_{2}-r\right)^{-\beta_{2}}$ and let $r \rightarrow b_{2}$, we obtain the following system of equations:

$$
\begin{aligned}
& \left(\cos \pi \alpha+c_{11}+\alpha c_{12}+\frac{\alpha(\alpha+1)}{2} c_{13}\right) \frac{g_{1}(0)}{b_{1}{ }^{\beta} \sin \pi \alpha} \\
& \quad+\left(d_{11}+\alpha d_{12}\right) \frac{g_{2}(0)}{b_{2}{ }^{\beta} \sin \pi \alpha}=0, \\
& \left(d_{21}+\alpha d_{22}\right) \frac{g_{1}(0)}{b_{1}{ }_{1} \sin \pi \alpha} \\
& \quad+\left(\cos \pi \alpha+c_{21}+\alpha c_{22}+\frac{\alpha(\alpha+1)}{2} c_{23}\right) \frac{g_{2}(0)}{b_{2}{ }^{\beta} \sin \pi \alpha}=0, \\
& \frac{g_{1}\left(b_{1}\right)}{b_{1}{ }^{\alpha}} \cot \pi \beta_{1}=0, \\
& \frac{g_{2}\left(b_{2}\right)}{b_{2}^{\alpha}} \operatorname{cot\pi \beta } \beta_{2}=0 .
\end{aligned}
$$

$g_{j}(0)$ and $g_{j}\left(b_{j}\right),(j=1,2)$, are non-zero constants which will be shown to be related to the stress intensity factors. Thus, (19.c) and (19.d) give the following characteristic equations to determine $\beta_{1}$ and $\beta_{2}$ :

$$
\cot \pi \beta_{1}=0, \quad \cot \pi \beta_{2}=0 \text {. }
$$

From (20) the values of $\beta_{1}$ and $\beta_{2}$ satisfying the condition $0<\operatorname{Re}\left(\beta_{j}\right)<1,(j=1,2)$, are found to be $\beta_{1}=\beta_{2}=1 / 2$, which 
is the well-known result.

On the other hand, since $\alpha \neq 0$ and $\alpha \neq 1$, for (19.a) and (19.b) to give a non-zero solution for $g_{j}(0)$, the determinant of the coefficients must vanish, giving the characteristic equation to determine $\alpha$ as follows:

$$
\begin{aligned}
D(\alpha) & \equiv\left(\cos \pi \alpha+c_{11}+\alpha c_{12}+\frac{\alpha(\alpha+1)}{2} c_{13}\right)\left(\cos \pi \alpha+c_{21}\right. \\
& \left.+\alpha c_{22}+\frac{\alpha(\alpha+1)}{2} c_{23}\right)-\left(d_{11}+\alpha d_{12}\right)\left(d_{21}+\alpha d_{22}\right)=0 .
\end{aligned}
$$

From (19) it is clear that the constants $g_{1}(0)$ and $g_{2}(0)$ are not independent and are related by

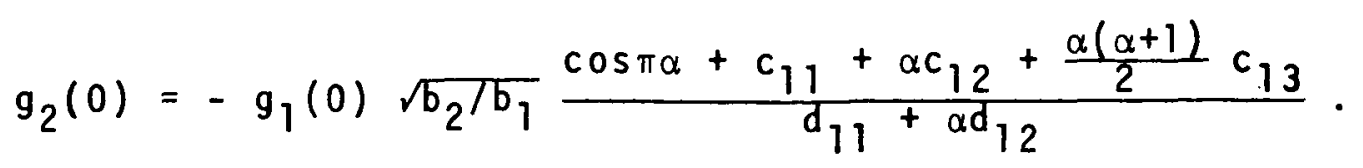

As will be pointed out later, the use of (22) will be necessary in order to obtain a unique solution for the integral equations (7).

In the special case of homogeneous medium, $m_{j} \rightarrow 1$, $k_{1}+k_{2}, \quad c_{j k}+0 \quad(j=1,2 ; k=1,2,3), \quad d_{11} \rightarrow 1, d_{12} \rightarrow 0$, $d_{21}=1, d_{22}+0$, the characteristic equation (20) becomes $\cos ^{2} \pi \alpha-1=0$, giving $\alpha=0$ as the acceptable root and $g_{2}(0) / \sqrt{b_{2}}=-g_{1}(0) / \sqrt{b_{1}}$. considering the definitions (5) and (12), this indicates that, as expected, at $r=0$ the derivative of the crack surface displacement becomes finite and continuous. 
In the problem of two bonded half planes containing a through crack perpendicular to the interface (Figure 1), the singular behavior of the solution at and around $r=0$ must be the same as that for two bonded quarter planes (see, for example, [11] and [12]). Defining the constants

$$
\begin{aligned}
& k_{1}=2\left(m_{1}-1\right), \quad k_{2}=m_{1}\left(1+k_{1}\right)-\left(1+k_{2}\right), \\
& k_{3}=m_{1}\left(1+k_{1}\right)+\left(1+k_{2}\right),
\end{aligned}
$$

after some manipulations (21) may be reduced to

$$
\begin{aligned}
& {\left[\left(k_{1}-k_{2}\right) \cos ^{2} \frac{\pi \alpha}{2}-k_{1}(\alpha-1)^{2}\right]^{2}} \\
& \quad+k_{3}^{2} \sin ^{2} \frac{\pi \alpha}{2} \cos ^{2} \frac{\pi \alpha}{2}-k_{2}^{2}(\alpha-1)^{2}=0 .
\end{aligned}
$$

(24) is identical to the characteristic equation found in [11] and [12] which indicates that the two solutions will have the same singular behavior .

In the special case of only one of the cracks terminating at the interface, i.e., for $b_{1}>a_{1}=0, b_{2}>a_{2}>0$, the kernels $k_{12}, k_{21}$ and $k_{22}$ in (7) will be bounded and will have no contribution to the singularities. For this problem the solution is of the form

\footnotetext{
As shown in [11] the weak power singularity $0<\operatorname{Re}(\alpha)<1$ as assumed in this paper is not the only possible singularity for two quarter planes. Theoretically it is possible to have a logarithmic singularity as well as bounded stresses. However, if one studies the conditions in detail, it is not difficult to show that for all practical material combinations, the power singularity is the only possibility.
} 


$$
f_{1}(s)=\frac{g_{1}(s)}{s^{\alpha_{1}}\left(b_{1}-s\right)^{\beta_{1}}}, \quad f_{2}(s)=\frac{g_{2}(s)}{\left(s-a_{2}\right)^{\alpha_{2}}\left(b_{2}-s\right)^{\beta_{2}}},
$$

$(25, a, b)$

and, following the procedure outlined above, the characteristic equations for $\alpha_{i}$ and $\beta_{i}(i=1,2)$ are found to be

$$
\begin{aligned}
& \cos \pi \alpha_{1}+c_{11}+\alpha c_{12}+\frac{\alpha(\alpha+1)}{2} c_{13}=0, \\
& \cot \pi \beta_{1}=0, \quad \cot \pi \alpha_{2}=0, \quad \cot \pi \beta_{2}=0 . \quad(26 . a-d)
\end{aligned}
$$

(26.a) is the equation found in [8] for the single crack and (26.b-d) give $\beta_{1}=\alpha_{2}=\beta_{2}=1 / 2$.

\section{STRESS INTENSITY FACTORS}

Using the results of the previous section it can be shown that the strength of the stress singularity at the crack tips, conventionally known as the stress intensity factor, is related to and can be evaluated from the density functions or the displacement derivatives $f_{j}(r),(j=1,2)$, defined by $(5)$. First, consider the case of two cracks imbedded into the adjacent homogeneous materials (i.e., $b_{j}>a_{j}>0, j=1,2$ ) for which the functions $f_{j}$ and the related holomorphic functions $G_{j}(z)$ may be expressed as

$$
f_{j}(s)=\frac{g_{j}(s)}{\left(s-a_{j}\right)^{\alpha_{j}}\left(b_{j}-s\right)^{\beta}},
$$




$$
\begin{aligned}
G_{j}(z)= & \frac{1}{\pi} \int_{a_{j}}^{b_{j}} \frac{f_{j}(s) d s}{s-z}=\frac{g_{j}\left(a_{j}\right) e^{\pi i \alpha_{j}}}{\left(b_{-j}-a_{-j}\right)^{\beta} j_{s i n \pi \alpha_{j}}} \frac{1}{\left(-z-a_{-j}\right)^{\alpha_{j}}} \\
& -\frac{g_{j}\left(b_{j}\right)}{\left(b_{j}-a_{j}\right)^{\alpha_{j}} j_{\text {sin } \pi \beta_{j}}} \frac{1}{\left(z-b_{j}\right)^{\beta_{j}}}+G_{o j}(z), \quad(j=1,2)
\end{aligned}
$$

where $\alpha_{j}=\beta_{j}=1 / 2$. We now note that in equations (7) the left hand sides are the expressions for $\left[\left(1+\kappa_{j}\right) / 2 \mu_{j}\right] \tau_{j \theta \theta}$ for $\left(0<r<a_{j}, b_{j}<r<\infty\right)$ as well as for $\left(a_{j}<r<b_{j}\right)$ as indicated in ( 7$)$. Thus, noting that the terms involving $k_{i j}$ are bounded, outside the cuts $G_{j}(r)$ directly gives $\tau_{j \theta \theta}$ on the real axes, from which the stress intensity factors may be readily evaluated. For example, at the end $r=b_{1}$ from (27) we obtain

$$
\begin{aligned}
k\left(b_{1}\right) & =\lim _{r \rightarrow b_{1}} \sqrt{2\left(r-b_{1}\right)} \tau_{1 \theta \theta}(r, \pi)=\frac{2 \mu_{1}}{\Gamma_{1}} \lim _{r \rightarrow b_{1}} \sqrt{2\left(r-b_{1}\right)} G_{1}(r) \\
& =-\frac{2 \mu_{1}}{1+k_{1}} \frac{\sqrt{2} g_{1}\left(b_{1}\right)}{\sqrt{b_{1}-a_{1}}}=-\frac{2 \mu_{1}}{1+k_{1}} \lim _{r \rightarrow b_{1}} \sqrt{2\left(b_{1}-r\right)} f_{1}(r) .
\end{aligned}
$$

The other expressions given in (10) follow from (27) in a simi1 ar way which, in terms of $g_{j}$, become

$$
\begin{aligned}
& k\left(b_{2}\right)=-\frac{2 \mu_{2}}{1+k_{2}} \frac{\sqrt{2} g_{2}\left(b_{2}\right)}{\sqrt{b_{2}-a_{2}}}, \\
& k\left(a_{j}\right)=\frac{2 \mu_{j}}{\sqrt{+k_{j}}} \frac{\sqrt{2} g_{j}\left(a_{j}\right)}{\sqrt{b_{j}-a_{j}}}, \quad(j=1,2)
\end{aligned}
$$

Consider now the second typical case, that is, a crack tip 
terminating at the interface (e.g., $b_{1}>a_{1}=0, b_{2}>a_{2}>0$ ). In this case the stress intensity factor will be defined as

$$
k_{1}(0)=\lim _{r \rightarrow 0} \sqrt{2} r^{\alpha} \tau_{2 \theta \theta}(r, 0)
$$

where $\alpha_{1}$ is obtained from $(26 . a)$ and $\tau_{2 \theta \theta}(r, 0)$ is given by $(7 . b)$. Since $b_{2}>a_{2}>0$, at $r=0$ the first and third terms on the left hand side of (7.b) will be bounded, and only the second term will contribute to the stress singularity. In the second term $a_{1}=0,0<s<b_{1}, r>0$, and $k_{21}(r, s)$ is given by (8.d). Hence, the asymptotic value of the integral for small $r$ may be obtained from $(8 . d)$ and $(18, a, b)$. Using the definition (30) and ignoring the bounded terms, we then obtain

$$
\begin{aligned}
& k_{1}(0)=\lim _{r \rightarrow 0} \sqrt{2} r^{\alpha} 1 \frac{2 \mu_{2}}{1+k_{2}} \frac{1}{\pi} \int_{0}^{b_{1}} \sum_{k=1}^{2} \frac{d_{2 k} r^{k-1}}{(s+r)^{k}} f_{1}(s) d s
\end{aligned}
$$

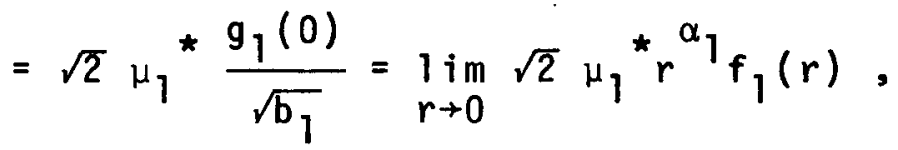

$$
\begin{aligned}
& \mu_{1}^{*}=\frac{2 \mu_{1}}{\Gamma_{\kappa_{1}}} \frac{d_{21}+\alpha d_{22}}{\sin \pi \dot{\alpha}} \\
& =m_{1} \mu_{1} \frac{\left(3-2 \alpha_{1}\right)\left(1+m_{1} \kappa_{1}\right)-\left(1-2 \alpha_{1}\right)\left(m_{1}+\kappa_{2}\right)}{\left(1+m_{1} \kappa_{1}\right)\left(m_{1}+k_{2}\right) \sin \pi \alpha_{1}} .
\end{aligned}
$$

Similarly for $b_{1}>a_{1}>0, b_{2}>a_{2}=0$, we find

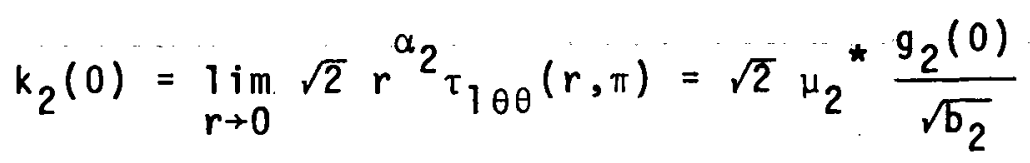

where $\mu_{2}{ }^{\star}$ is obtained from (31.b) by interchanging the indices 
1 and 2 in $\mu_{j}, m_{j}, k_{j},(j=1,2)$, and replacing $\alpha_{1}$ by $\alpha_{2}$ which, in turn, is obtained from $(26 . a)$ by substituting $c_{2 k}$ for $c_{1 k}$, $(k=1,2,3)$. It should be noted that the bielastic constant $\mu_{1}{ }^{*}$ is identical to $\mu^{*}$ given in [8] which was obtained by a different method. Needless to say, in this case for the remaining three end points the expressions given by (28) and (29) will remain valid.

For the third case in which the crack goes through the interface, i.e., for $a_{1}=a_{2}=0, b_{1}>0, b_{2}>0$, we define the normal and the shear components of the stress intensity factor as follows*:

$$
\begin{aligned}
k_{\theta}=\lim _{r \rightarrow 0} r^{\alpha} \tau_{j \theta \theta}(r, \pi / 2), \quad k_{r} & =\lim _{r \rightarrow 0} r^{\alpha} \tau_{j r \theta}(r, \pi / 2), \\
(j & =1 \text { or } 2) .
\end{aligned}
$$

Thus, to obtain $k_{\theta}$ and $k_{r}$ the expressions for the interface stresses are needed. These expressions may be obtained as definite integrals with the density functions $f_{j},(j=1,2)$,

In this case, the asymptotic expressions of the stresses for small values of $r$ and for $0<\theta<\pi$ are of the form

$\tau_{i j}(r, \theta)=\frac{k(0)}{r^{\alpha}} h_{i j}(\theta)+0\left(r^{\alpha^{1}}\right),\left(i, j=r, \theta ; \operatorname{Re}(\alpha)>\operatorname{Re}\left(-\alpha^{1}\right)\right)$,

where $h_{\theta \theta}$ and $h_{r \theta}$ are bounded and continuous in $[0, \pi]$, including at $\theta=\pi / 2$, and the bounded function $h_{r r}$ has a discontinuity at $\theta=\pi / 2$. Here, because of the physical importance of the interface and simplicity of the calculations, the stress intensity factors are defined in terms of the normal and shear stresses along the interface, i.e., $k_{\theta}=k(0) h_{\theta \theta}(\pi / 2)$, $k_{r}=k(0) h_{r \theta}(\pi / 2)$. As the results of this section show, within a multiplicative constant, $k(0)$ is nothing but the constant $g_{1}(0)$ (which is related to $g_{2}(0)$ through (22)). 
and the appropriate kernels which are evaluated from the original Mellin Transform solution (see [8]). After some manipulations, the components of the stress vector at the interface are found to be

$$
\begin{aligned}
& \tau_{1 \theta \theta}\left(r, \frac{\pi}{2}\right)=\tau_{2 \theta \theta}\left(r, \frac{\pi}{2}\right)=\frac{1}{\pi} \int_{0}^{b_{1}}\left[\frac{A_{1} s}{s^{2}+r^{2}}+\frac{A_{2} s\left(3 r^{2}-s^{2}\right)}{\left(s^{2}+r^{2}\right)^{2}}\right] f_{1}(s) d s \\
& +\frac{1}{\pi} \int_{0}^{b}\left[\frac{A_{3} s}{s^{2}+r^{2}}+\frac{A_{4} s\left(3 r^{2}-s^{2}\right)}{\left(s^{2}+r^{2}\right)^{2}}\right] f_{2}(s) d s \\
& \tau_{1 r \theta}\left(r, \frac{\pi}{2}\right)=\tau_{2 r \theta}\left(r, \frac{\pi}{2}\right)=\frac{1}{\pi} \int_{0}^{b_{1}}\left[\frac{B_{1} r}{s^{2}+r^{2}}+\frac{B_{2} r\left(r^{2}-3 s^{2}\right)}{\left(s^{2}+r^{2}\right)^{2}}\right] f_{1}(s) d s \\
& +\frac{1}{\pi} \int_{0}^{b_{2}}\left[\frac{B_{3} r}{s^{2}+r^{2}}+\frac{B_{4} r\left(r^{2}-3 s^{2}\right)}{\left(s^{2}+r^{2}\right)^{2}}\right] f_{2}(s) d s \\
& A_{1}=\frac{\mu_{2}}{m_{1}+k_{2}}, \quad A_{2}=-\frac{\mu_{2}}{1+m_{1} k_{1}}, \quad A_{3}=-\frac{\mu_{1}}{m_{2}+\kappa_{1}}, \quad A_{4}=\frac{\mu_{1}}{1+m_{2} k_{2}}, \\
& B_{1}=\frac{\mu_{2}\left(1+m_{1} k_{2}\right)}{\left(1+m_{1} k_{1}\right)\left(m_{1}+k_{2}\right)}, \quad B_{2}=\frac{\mu_{2}\left(m_{1}+\kappa_{2}\right)}{\left(1+m_{1} k_{1}\right)\left(m_{1}+k_{2}\right)}, \\
& B_{3}=\frac{\mu_{1}\left(1+m_{2} k_{1}\right)}{\left(1+m_{2} k_{2}\right)\left(m_{2}+k_{1}\right)}, \quad B_{4}=\frac{\mu_{1}\left(m_{2}+\kappa_{1}\right)}{\left(1+m_{2} k_{2}\right)\left(m_{2}+k_{1}\right)} .
\end{aligned}
$$

The kernels in (34) can easily be expressed as the sum of terms containing

$$
\frac{1}{s+i r}, \frac{1}{s-i r}, \frac{i r}{(s+i r)^{2}}, \frac{i r}{(s-i r)^{2}} \text {. }
$$

It is clear that since $r>0$, at $z= \pm$ ir the functions $G_{j}(z)$ 
defined by (13) will be holomorphic and, following the same procedure which led to (18) in the previous section, the integrats in (-34) may be evaluated-in terms of G(z) and-its derivative (at $z= \pm i r)$. For example,

$$
\begin{aligned}
& \frac{1}{\pi} \int_{0}^{b_{1}} \frac{s f_{f}(s) d s}{s^{2}+r^{2}}=\frac{1}{2 \pi} \int_{0}^{b_{1}}\left(\frac{1}{s+i r}+\frac{1}{s-i r}\right) f_{1}(s) d s \\
& =\frac{1}{2}\left(e^{\pi i \alpha / 2}+e^{-\pi i \alpha / 2}\right) \frac{g_{1}(0)}{b_{1}{ }^{\beta}{ }_{\sin \pi \alpha}} \frac{1}{r^{\alpha}}+F_{1}(r) \\
& =\frac{g_{1}(0)}{2 b_{1}{ }^{\beta_{1}} \sin \frac{\pi \dot{\alpha}}{2}} \frac{1}{r^{\alpha}}+F_{1}(r) \text {, } \\
& \frac{1}{\pi} \int_{0}^{b_{1}} \operatorname{ir}\left(\frac{1}{(s+i r)^{2}}-\frac{1}{(s-i r)^{2}}\right) f_{1}(s) d s \\
& =\frac{\alpha g l(0)}{b_{1}{ }_{1} \sin \frac{\pi \alpha}{2}} \frac{1}{r^{\alpha}}+F_{2}(r) \text {, }
\end{aligned}
$$

where $F_{1}$ and $F_{2}$ are bounded functions. Carrying out the necessary manipulations, from (33-37). we obtain

$$
\begin{aligned}
& k_{\theta}=\frac{1}{2 \sin \frac{\pi \alpha}{2}}\left\{\frac{g_{1}(0)}{\sqrt{b_{1}}}\left[A_{1}-(1-2 \alpha) A_{2}\right]-\frac{g_{2}(0)}{\sqrt{b_{2}}}\left[A_{3}-(1-2 \alpha) A_{4}\right]\right\}, \\
& k_{r}=\frac{1}{2 \cos \frac{\pi \alpha}{2}}\left\{\frac{g_{1}(0)}{\sqrt{b}}\left[B_{1}-(1-2 \alpha) B_{2}\right]-\frac{g_{2}(0)}{\sqrt{b_{2}}}\left[B_{3}-(1-2 \alpha) B_{4}\right]\right\}, \\
& (38, a, b)
\end{aligned}
$$

where the constants $g_{1}(0)$ and $g_{2}(0)$ are linearly related through (22) and the constants $A_{j}$ and $B_{i}(i=1, \ldots, 4)$ are given by $(35)$. 


\section{CRACK SURFACE DISPLACEMENTS}

The crack surface displacements $u_{1 \theta}$ and $u_{2 \theta}$ are related to the density functions $f_{1}$ and $f_{2}$ through (5). Hence, noting that $u_{j \theta}=0$ for $r>b_{j},(j=1,2)$, after evaluating $f_{1}$ and $f_{2}$ the displacements may be obtained from

$$
\begin{aligned}
& u_{1 \theta}(r, \pi-0)=\frac{1}{2} \int_{r}^{b_{1}} f_{1}(s) d s, \\
& u_{2 \theta}(r,+0)=-\frac{1}{2} \int_{r}^{b_{2}} f_{2}(s) d s .
\end{aligned}
$$

6. SOLUTION OF THE INTEGRAL EQUATIONS AND NUMERICAL RESULTS

In deriving the integral equations ( 7 ), instead of the conditions (2.c) and (4.c) the conditions (6.a,b) are used; that is, the problem is formulated on the assumption that outside the cuts $\left(a_{j}, b_{j}\right)$, the displacement derivatives $f_{j}$ rather than the displacements $u_{j \theta},(j=1,2)$, are zero. Thus, in the case of nonintersecting cracks (i.e., for $a_{1}>0, a_{2}>0$, or $\left.a_{1}=0, a_{2}>0\right)$, the integral equations (7) must be solved under the single-valuedness conditions (6.c,d). Referring to [10], since the general solution of the system of singular integral equations [7] will contain two arbitrary constants... the two additional conditions $(6, c, d)$ are needed for a unique solution. On the other hand for the intersecting cracks, that is, for $a_{1}=0=a_{2}$, there is only one single-valuedness condition, namely, $-u_{1 \theta}(0, \pi-0)=u_{2 \theta}(0,+0)$, or 


$$
\int_{0}^{b_{1}} f_{1}(s) d s=\int_{0}^{b_{2}} f_{2}(s) d s
$$

The second condition which is necessary for a unique solution is provided by (22) relating $g_{1}(0)$ and $g_{2}(0)$, or the functions $f_{1}$ and $f_{2}$ at $r=0$.

For $a_{1}=a_{2}=0$, even though the special cases of the resulting system of singular integral equations with generalized Cauchy kernels (7) have been considered in [13] and [14], there is no known method which can be used to regularize the integral equations of this type. To solve these equations we first normalize the intervals $\left(a_{j}, b_{j}\right),(j=1,2)$, through the following change in variables:

$$
\begin{array}{r}
\xi_{1}=\frac{2 s-\left(b_{1}+a_{1}\right)}{b_{1}-a_{1}}, \quad \xi_{2}=\frac{2 s-\left(b_{2}+a_{2}\right)}{b_{2}-a_{2}}, \\
\quad\left(a_{j}<s<b_{j},-1<\xi_{j}<1, j=1,2\right) .
\end{array}
$$

Next we let

$$
f_{j}(s)=\frac{g_{j}(s)}{(s-a)^{\alpha}{ }^{\alpha}\left(b_{j}-s\right)^{\beta}}=\frac{\phi_{j}\left(\xi_{j}\right)}{\left(1+\xi_{j}\right)^{\alpha}{ }^{\alpha}\left(1-\xi_{j}\right)^{\beta}},
$$

giving

$$
g_{j}(s)=\left(\frac{b_{j}^{-a_{j}}}{2}\right)^{\alpha_{j}+\beta} \phi_{j}\left(\xi_{j}\right) .
$$

Also defining 


$$
\begin{aligned}
t_{j}= & \frac{2 r-\left(b_{j}+a_{j}\right)}{b_{j}-a_{j}}, \quad P_{j}\left(t_{j}\right)=p_{j}(r), \\
& \left(a_{j}<r<b_{j},-1<t_{j}<1, \quad j=1,2\right),
\end{aligned}
$$

and suppressing the subscripts in $\xi_{j}$ and $t_{j}$, the system of integral equations (7) may be expressed as

$$
\begin{aligned}
\frac{1}{\pi} \int_{-1}^{1} \sum_{1}^{2}\left[\frac{\delta_{i j}}{\xi-t}+k_{i j}(t, \xi)\right] \phi_{j}(\xi) w_{j}(\xi) d \xi=-\frac{1+k_{i}}{2 \mu_{i}} p_{j}(t), \\
\quad(-1<t<1),
\end{aligned}
$$

where

$$
w_{j}(\xi)=(1+\xi)^{-\alpha_{j}}(1-\xi)^{-\beta_{j}}, \quad(j=1,2)
$$

are the fundamental functions of the system, and the kernels $k_{i j}$ may be obtained from $k_{i j}$ by means of appropriate transformations. Now, observing that the fundamental functions $w_{j}(\xi)$ of the system of integral equations are weights of the Jacobi polynomials $P_{n}\left(-\beta_{j},-\alpha_{j}\right)(\xi)$, the equations may be solved numerically for the unknown functions $\phi_{j}$ by using the Gauss-Jacobi integration formulas described in [15]. After evaluating $\phi_{j}$, the values of $g_{j}$ at the end points, which are needed to calculate the stress intensity factors, may be obtained from (43) in terms of $\phi_{j}( \pm 1)$.

For the material pairs Aluminum-Epoxy some of the calculated results for the plane strain case are shown in Tables 3-5 and Figures 4-13. For $b_{1} \rightarrow 0$ or $b_{2} \rightarrow 0$ (i.e., for the case of a single crack terminating at the interface), the results 
of [8] are obtained as the limiting values. In the numerical examples given in this paper it is assumed that the composite medium ${ }^{-i} s^{-r o a d e} d^{-}$paralte ${ }^{-}$to the ${ }^{-}$interface and away from the crack region in such a way that the stresses $p_{1}(r)$ and $p_{2}(r)$ are approximately constant. Thus, in the singular part of the problem considered in this paper, the crack surface tractions will be constant pressures $-p_{1}$ and $-p_{2}$ which are related through (1).

In the example shown in Table 3 and Figures 4 and 5 , the material 1 is aluminum, material 2 is epoxy, one of the crack tips $b_{1}$ is fixed at a unit distance from the interface, and $b_{2}$ varies between 0 and $2 b_{1}$. In Figure 4 as well as 6 and 8 the constants $k_{1}$ and $k_{2}$ refer to $k\left(b_{1}\right)$ and $k\left(b_{2}\right)$, respectively.

Table 3. The Stress Intensity Factors for Through Crack, $a_{1}=a_{2}=0$. Material 1: Aluminum, Material 2: Epoxy $\left(\nu_{1}=0.3, \nu_{2}=0.35, E_{1} / E_{2}=22.447\right)$; $b_{1}=1=$ constant, $l=\left(b_{1}+b_{2}\right) / 2, b_{2}$ variable, .$\left(\beta_{1}=\beta_{2}=0.5, \alpha=0.273692\right)$.

\begin{tabular}{|c|c|c|c|c|}
\hline$b_{2} / b_{1}$ & $k\left(b_{1}\right) / p_{1} \sqrt{l}$ & $k\left(b_{2}\right) / p_{2} \sqrt{l}$ & $k_{\theta} / p_{1} e^{\alpha}$ & $k_{r} / p_{1} l^{\alpha}$ \\
\hline 0.00 & $1.3552^{*}$ & $+\infty$ &.$--\infty$ & $+\infty$ \\
0.05 & 1.40374 & 4.36065 & -0.22477 & 0.08215 \\
0.25 & 1.33240 & 2.13917 & -0.08113 & 0.03184 \\
0.50 & 1.23789 & 1.55615 & -0.05906 & 0.02158 \\
0.75 & 1.15887 & 1.31166 & -0.04636 & 0.01694 \\
1.00 & 1.09312 & 1.17874 & -0.03839 & 0.01403 \\
1.25 & 1.03767 & 1.09693 & -0.03266 & 0.01194 \\
1.50 & 0.99020 & 1.04261 & -0.02822 & 0.01032 \\
1.75 & 0.94902 & 1.00462 & -0.02462 & 0.00900 \\
2.00 & 0.91286 & 0.97701 & -0.02160 & 0.00789 \\
\hline
\end{tabular}

*Taken from Reference [8]. 
Table 4. The Stress Intensity Factors for Through Crack, $a_{1}=a_{2}=0$. Material 1: Aluminum, Material 2: Epoxy; $2 l=b_{1}+b_{2}=2=$ constant, $c=\left(b_{2}-b_{1}\right) / 2$ variable, $\left(\beta_{1}=\beta_{2}=0.5, \alpha=0.27369 .2\right)$.

\begin{tabular}{|c|c|c|c|c|}
\hline$c / l$ & $k\left(b_{1}\right) / p_{1} \sqrt{l}$ & $k\left(b_{2}\right) / p_{2} \sqrt{l}$ & $k_{\theta} / p_{1} l^{\alpha}$ & $k_{r} / p_{1} l^{\alpha}$ \\
\hline-1.00 & $1.3552^{*}$ & $\rightarrow \infty$ & $\rightarrow-\infty$ & $+\infty$ \\
-0.95 & 1.40333 & 5.69903 & -0.34124 & 0.12472 \\
-0.75 & 1.37532 & 2.76781 & -0.11960 & 0.04371 \\
-0.50 & 1.29929 & 1.87137 & -0.07423 & 0.02713 \\
-0.25 & 1.20448 & 1.43765 & -0.05310 & 0.01941 \\
0.00 & 1.09312 & 1.17874 & -0.03839 & 0.01403 \\
0.25 & 0.96213 & 1.01590 & -0.02575 & 0.00941 \\
0.50 & 0.80281 & 0.91834 & -0.01293 & 0.00473 \\
0.75 & 0.59068 & 0.87340 & 0.00313 & -0.00114 \\
0.95 & 0.29854 & 0.87519 & 0.02903 & -0.01061 \\
1.00 & $\rightarrow 0$ & $0.8827^{*}$ & $\rightarrow \infty$ & $\rightarrow-\infty$ \\
\hline
\end{tabular}

*Taken from Reference [8].

Table 5. The Stress Intensity Factors for Through Crack, $a_{1}=a_{2}=0$. Material 1: Epoxy, Material 2: Aluminum; $b_{1}=1=$ constant, $\ell=\left(b_{1}+b_{2}\right) / 2$, $b_{2}$ variable, $\left(\alpha=0.273692, \beta_{1}=\beta_{2}=0.5\right)$.

\begin{tabular}{|c|c|c|c|c|}
\hline$b_{2} / b_{1}$ & $k\left(b_{1}\right) / p_{1} \sqrt{l}$ & $k\left(b_{2}\right) / p_{2} \sqrt{l}$ & $k_{\theta} / p_{1} l^{\alpha}$ & $k_{r} / p_{1} l^{\alpha}$ \\
\hline 0.00 & $0.8827^{*}$ & +0 & $+\infty$ & $+\infty$ \\
0.05 & 0.87141 & 0.39001 & 0.44554 & 0.16283 \\
0.25 & 0.89435 & 0.72689 & -0.16129 & -0.05895 \\
0.50 & 0.97701 & 0.91286 & -0.48485 & -0.17720 \\
0.75 & 1.07647 & 1.02106 & -0.69736 & -0.25487 \\
1.00 & 1.17874 & 1.09312 & -0.86170 & -0.31493 \\
1.25 & 1.27901 & 1.14476 & -0.99895 & -0.36509 \\
1.50 & 1.37559 & 1.18357 & -1.11885 & -0.40891 \\
1.75 & 1.46797 & 1.21377 & -1.22669 & -0.44832 \\
2.00 & 1.55615 & 1.23789 & -1.32567 & -0.48449 \\
\hline
\end{tabular}

*Taken from Reference [8]. 


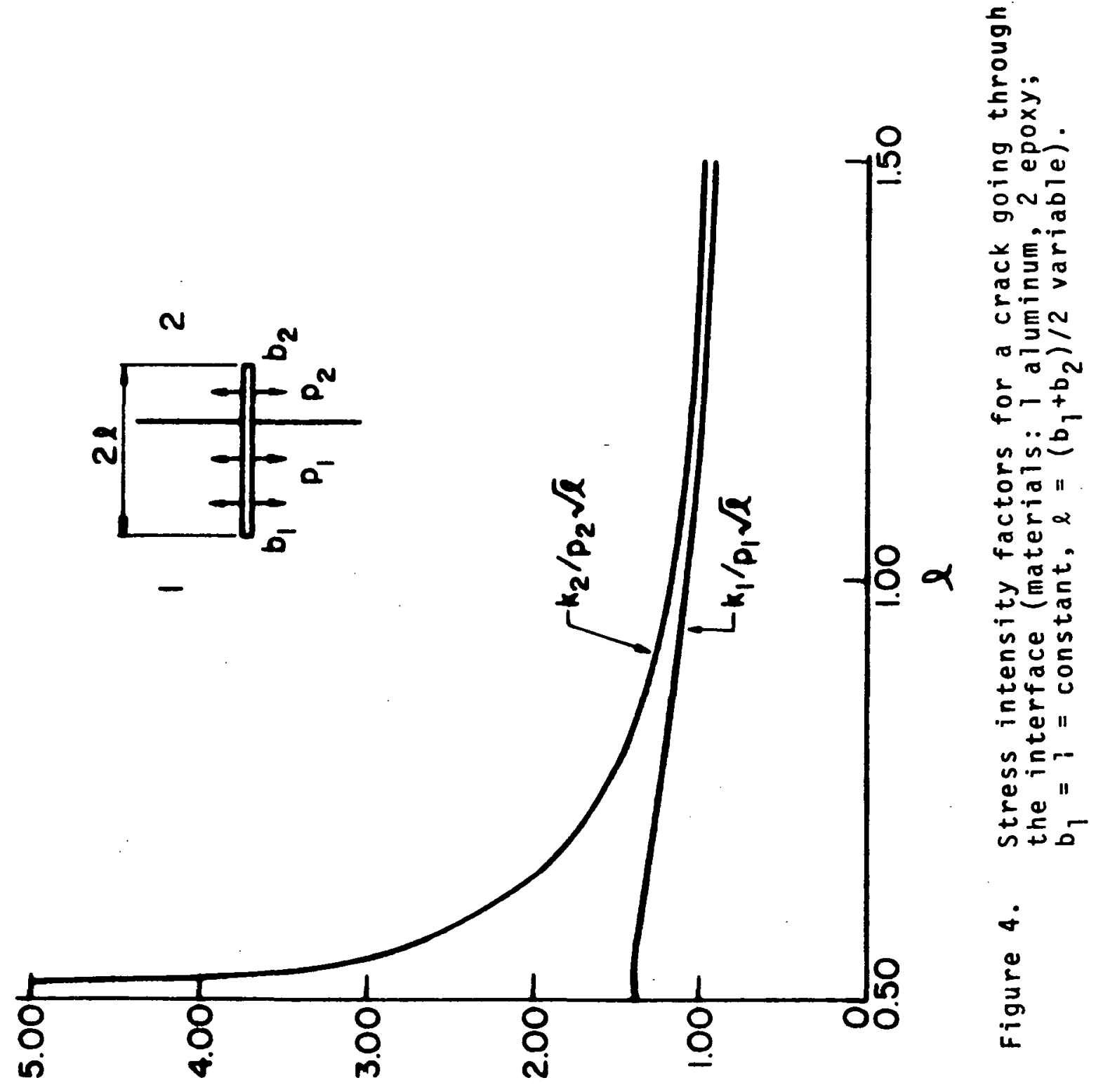




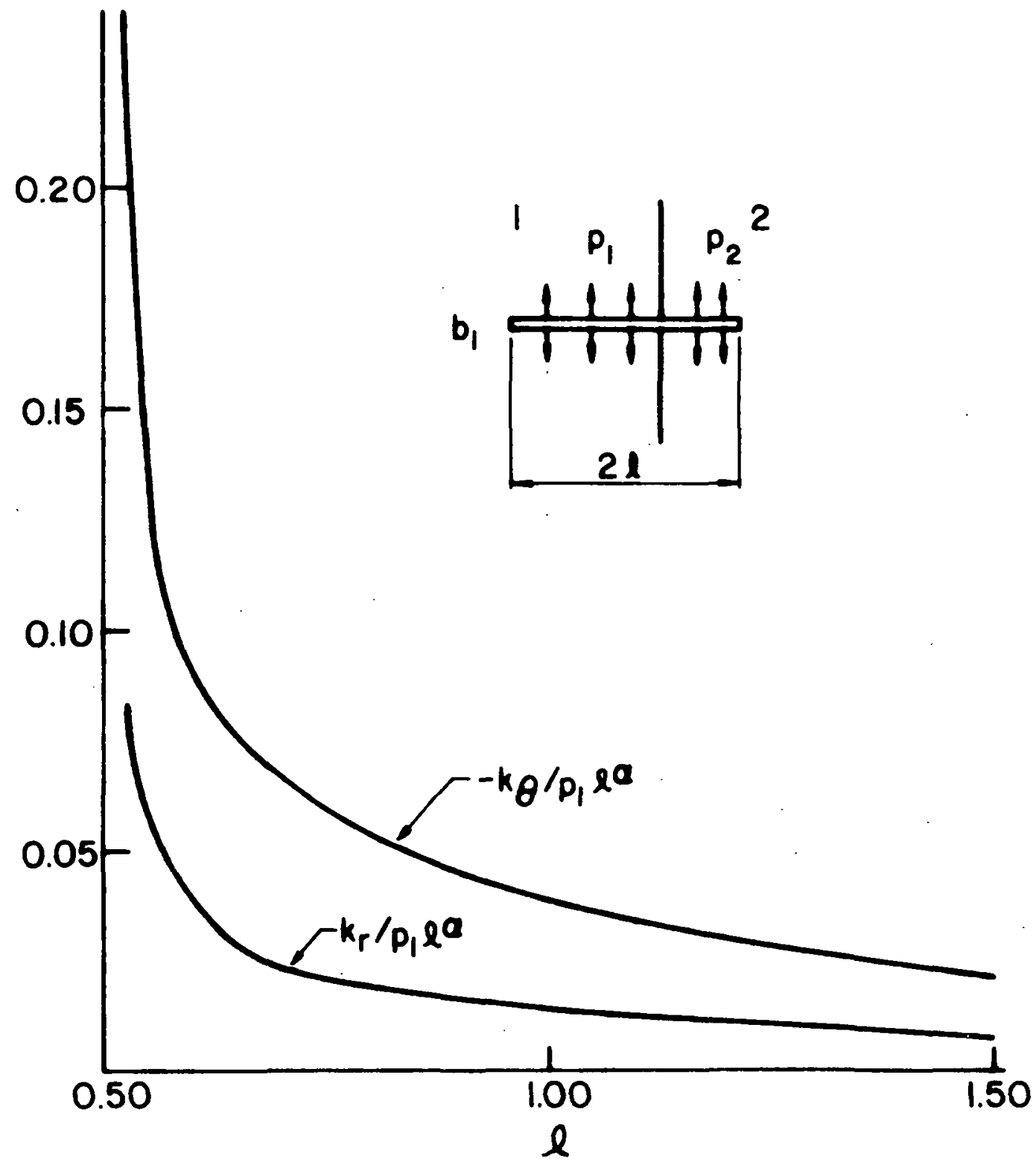

Figure 5. Stress intensity factors for normal and shear stresses on the interface for the through crack (materials: 1 aluminum, 2 epoxy; $b_{1}=$ constant). 
Table 4 and Figures 6 and 7 show the results for the same material combination. In this second example it is assumed that the total length $2 l=b_{1}+b_{2}$ of the crack is constant, and the distance $c=\left(b_{2}-b_{1}\right) / 2$ from its center to the interface is variable. The stress intensity factors for the case of epoxyaluminum are shown in Table 5 and Figures 8 and 9.

The tables also show the limiting values of the stress intensity factors for $b_{2} \rightarrow 0$ and $c+ \pm 1$. These limiting values are obtained by observing that in the case of a through crack (i.e., for $\left.b_{j}>a_{j}=0,(j=1,2)\right)$, for the material pair aluminum-epoxy (21) gives the power of the stress singularity as $\alpha=0.2737$. On the other hand, for the limiting case of a crack tip terminating at the interface, i.e., for $b_{1}=0$ or $b_{2}=0$, and for the material pair under consideration, from (26.a) the power $\alpha_{1}$ of the stress singularity is calculated to be :

$$
\begin{aligned}
& \alpha_{1}=0.8258, \text { crack in aluminum, } \\
& \alpha_{1}=0.3381, \text { crack in epoxy. }
\end{aligned}
$$

Thus, for example, in the example shown in Table 3 and Figures 4 and 5 , as $b_{2} \rightarrow 0$ the powers of singularity become $\alpha+\alpha_{1}$, $\beta_{2} \rightarrow \alpha_{1}$. Therefore, for this limiting case, since $\alpha_{1}=0.8258$ $>\alpha=0.2737$ and $\alpha_{1}>\beta_{2}=0.5$, at $r=0$ the stresses would have a stronger singularity and consequently the stress intensity factors $k_{\theta}$ and $k_{r}$, which are defined on the basis of $r^{-\alpha}$, and $k\left(b_{2}\right)$, which is defined on the basis of $r^{-0.5}$, would all go to infinity. Similar arguments apply to the limiting values 


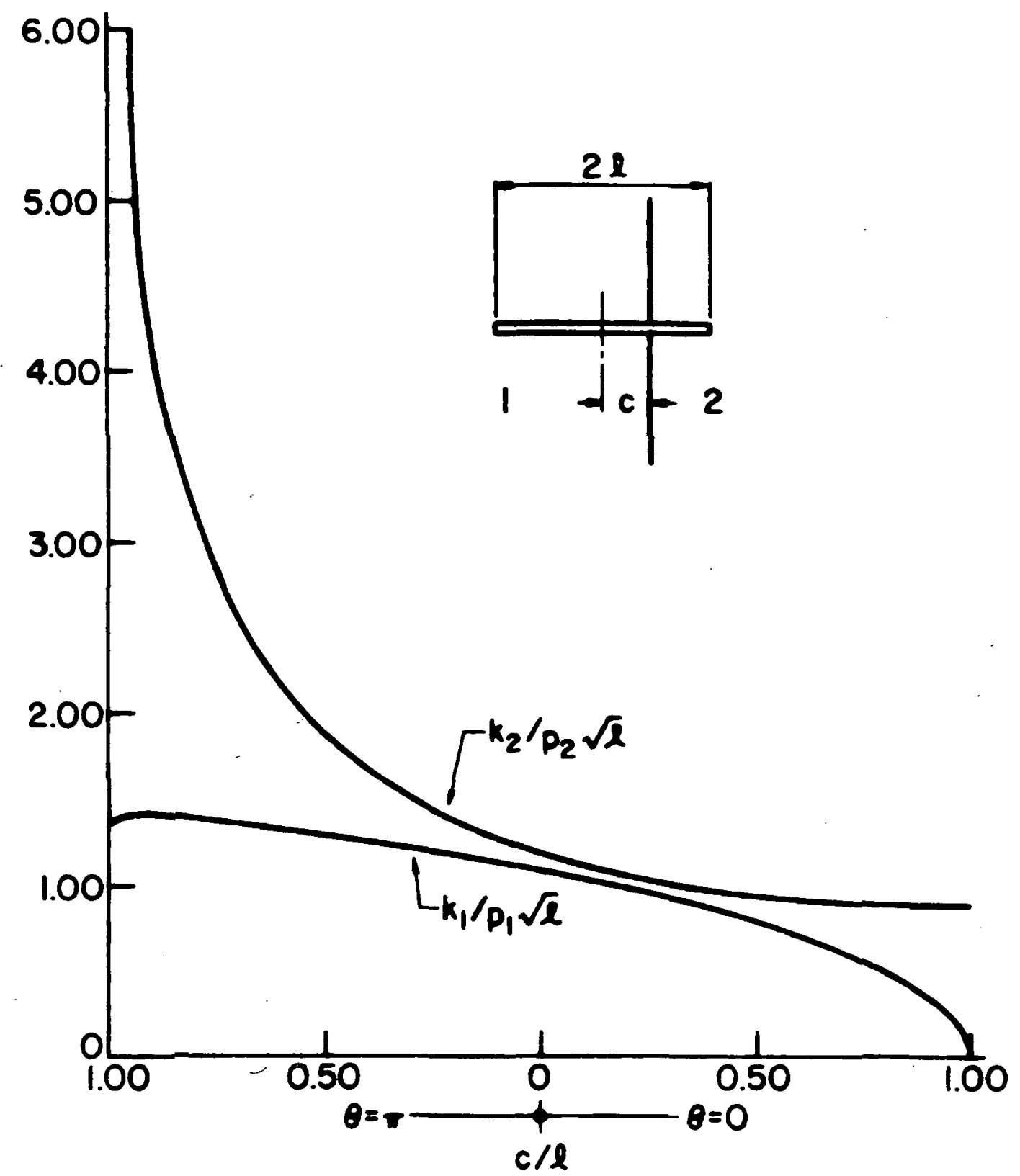

Figure 6. Stress intensity factors for through crack (materials: 1 aluminum, 2 epoxy; $2 l=2=$ constant, c variable). 


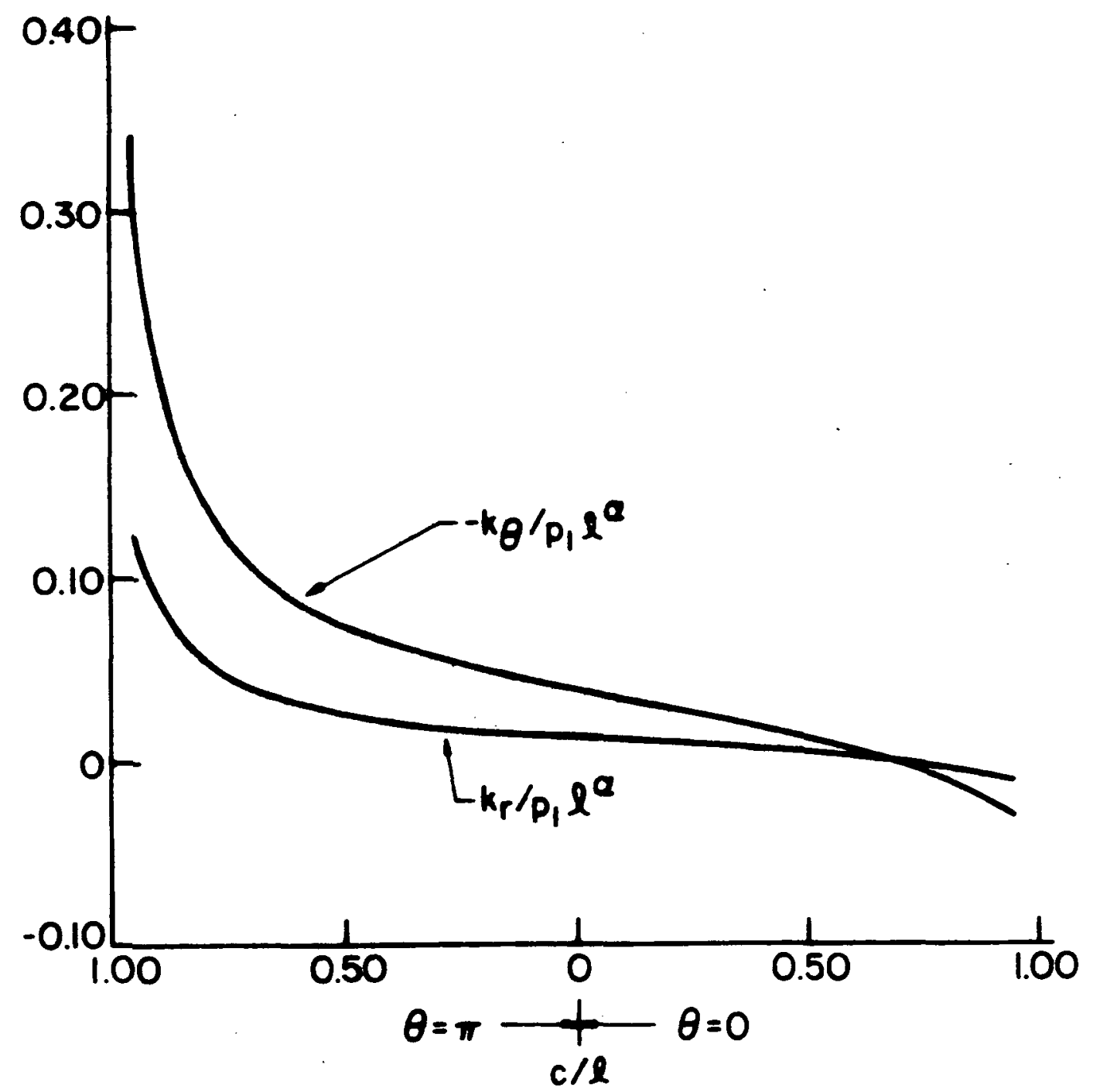

Figure 7. Stress intensity factors for normal and shear stresses on the interface for through crack (materials: 1 aluminum, 2 epoxy; $2 l=2=$ constant, $c$ variable). 


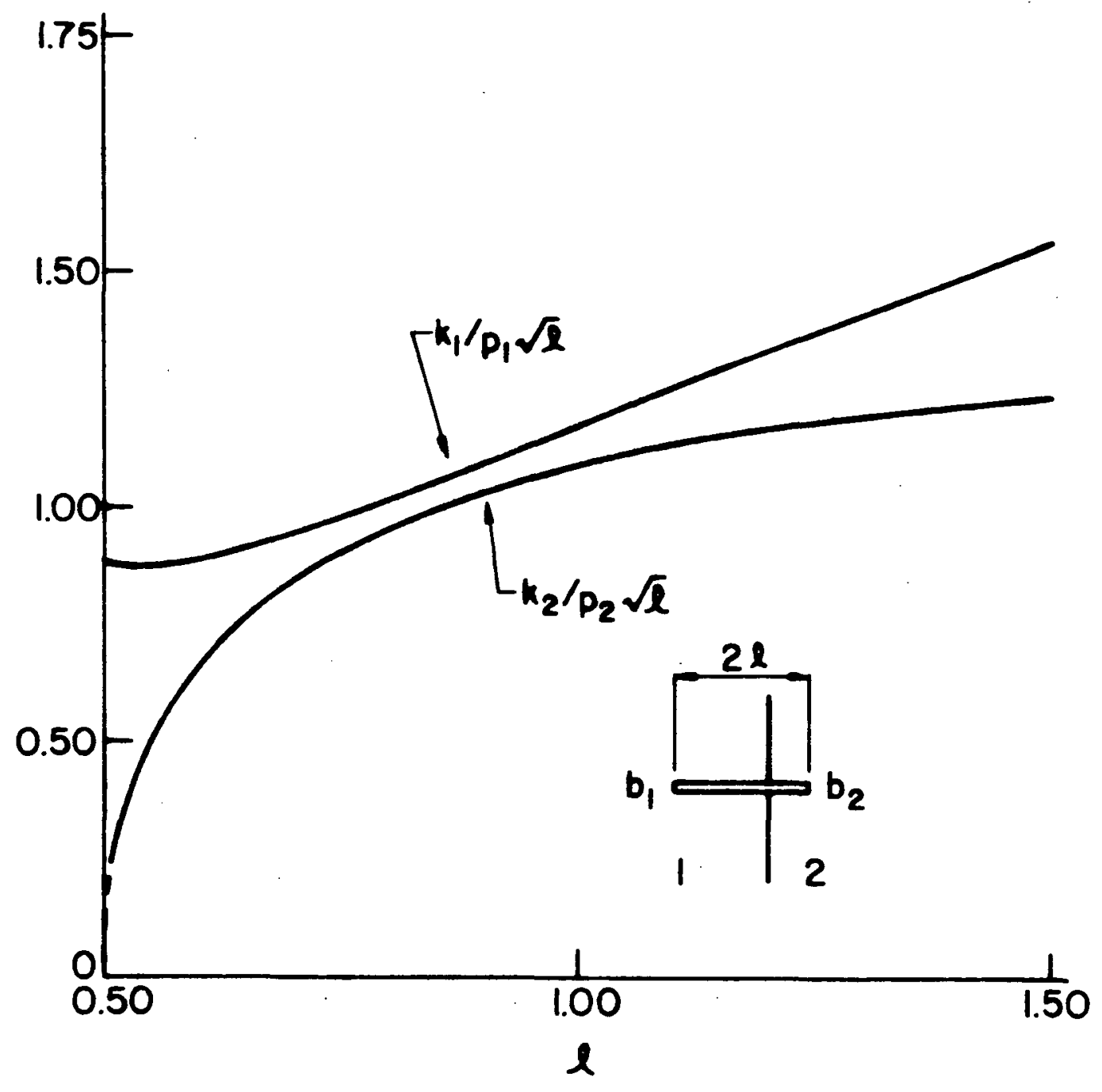

Figure 8. Stress intensity factors for through crack (materials: 1 epoxy, 2 aluminum; $b_{1}=1=$ constant, $\ell$ variable). 


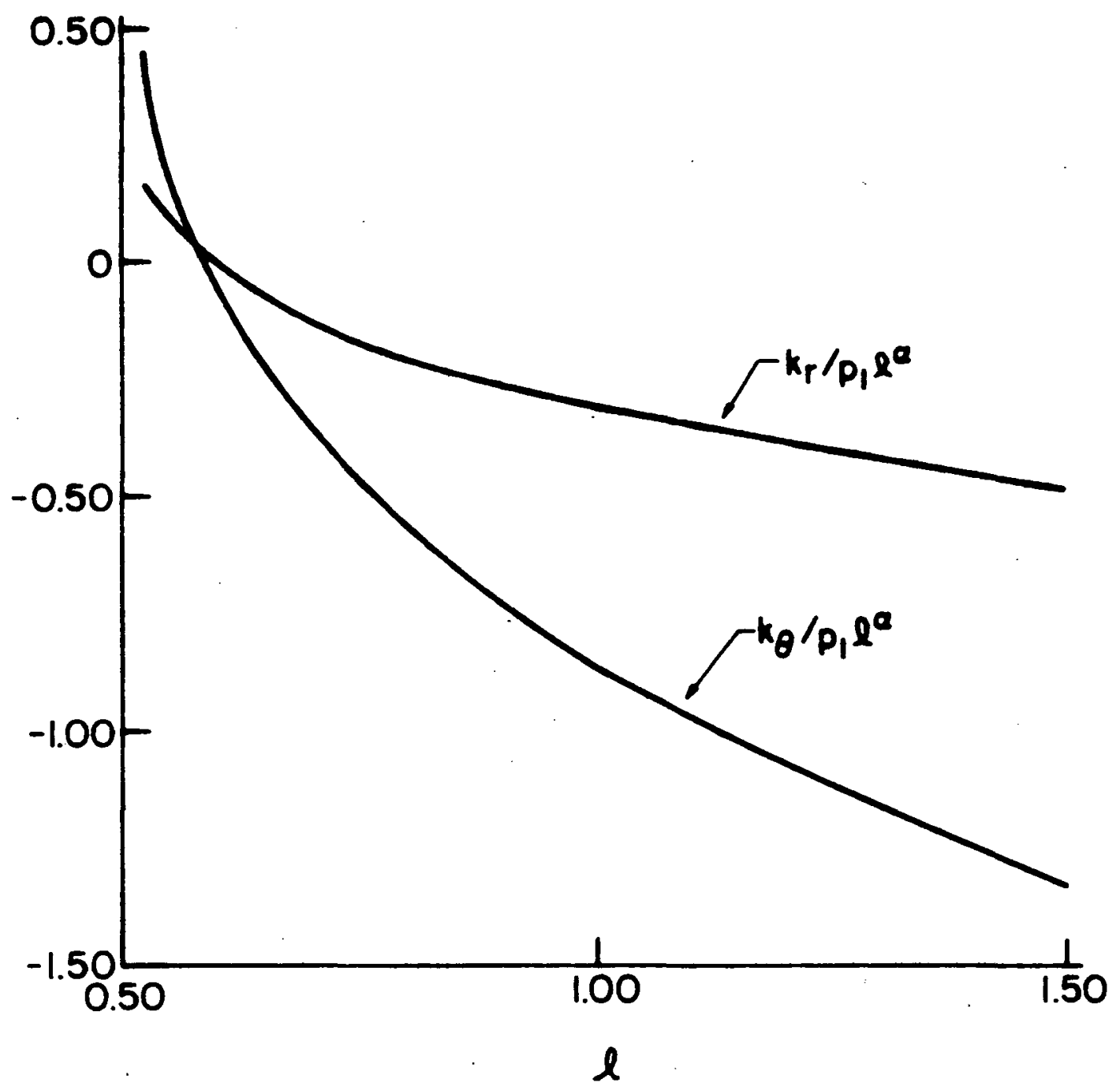

Figure 9. Stress intensity factors for normal and shear stresses on the interface for through crack (materials: 1 epoxy, 2 aluminum; $b_{1}=1=$ constant, $\ell$ variable). 
shown in Tables 4 and 5 .

From Tables $3-5$ and Figures $5,7,9$ it is seen that the normal component of the stress intensity factor, $k_{\theta}$, (hence, the normal component of the bonding stress along the interface in the neighborhood of $r=0$ ) may be positive or negative depending on the relative penetration depths $b_{1}$ and $b_{2}$ of the crack into the adjacent materials. As the ratio $b_{2} / b_{1}$ increases, $k_{\theta}$ increases if $\mu_{1}>\mu_{2}$ (Figures 5,7 ), and decreases if $\mu_{1}<\mu_{2}$ (Figure 9).

Figure 10 shows a sample distribution of the density functions $f_{1}$ and $f_{2}$ defined by

$$
\begin{array}{ll}
f_{1}(s)=F_{1}\left(\xi_{1}\right), & \left(0<s<b_{1},-1<\xi_{1}<1\right), \\
f_{2}(s)=F_{2}\left(\xi_{2}\right), & \left(0<s<b_{2},-1<\xi_{2}<1\right) .(48 . a, b)
\end{array}
$$

The calculated values shown in the figure correspond to the example given in Table 3 with $b_{2} / b_{1}=0.05$ (i.e., material 1 is aluminum, material 2 is epoxy). The important feature of the distribution of the density functions $f_{1}$ and $f_{2}$ is that at $r=0$, because of the common $r^{-\alpha}$ type singularity, they become infinite, $-f_{1}$ and $f_{2}$ having the same sign (i.e., at $r=0$ the derivative of the crack surface displacement is discontinuous and unbounded).

Sample results for the crack surface displacement calculated from $(39)$ are shown in Figures 11-13, where the variables are again the normalized quantities $\xi_{1}$ and $\xi_{2}$ defined by (41). 


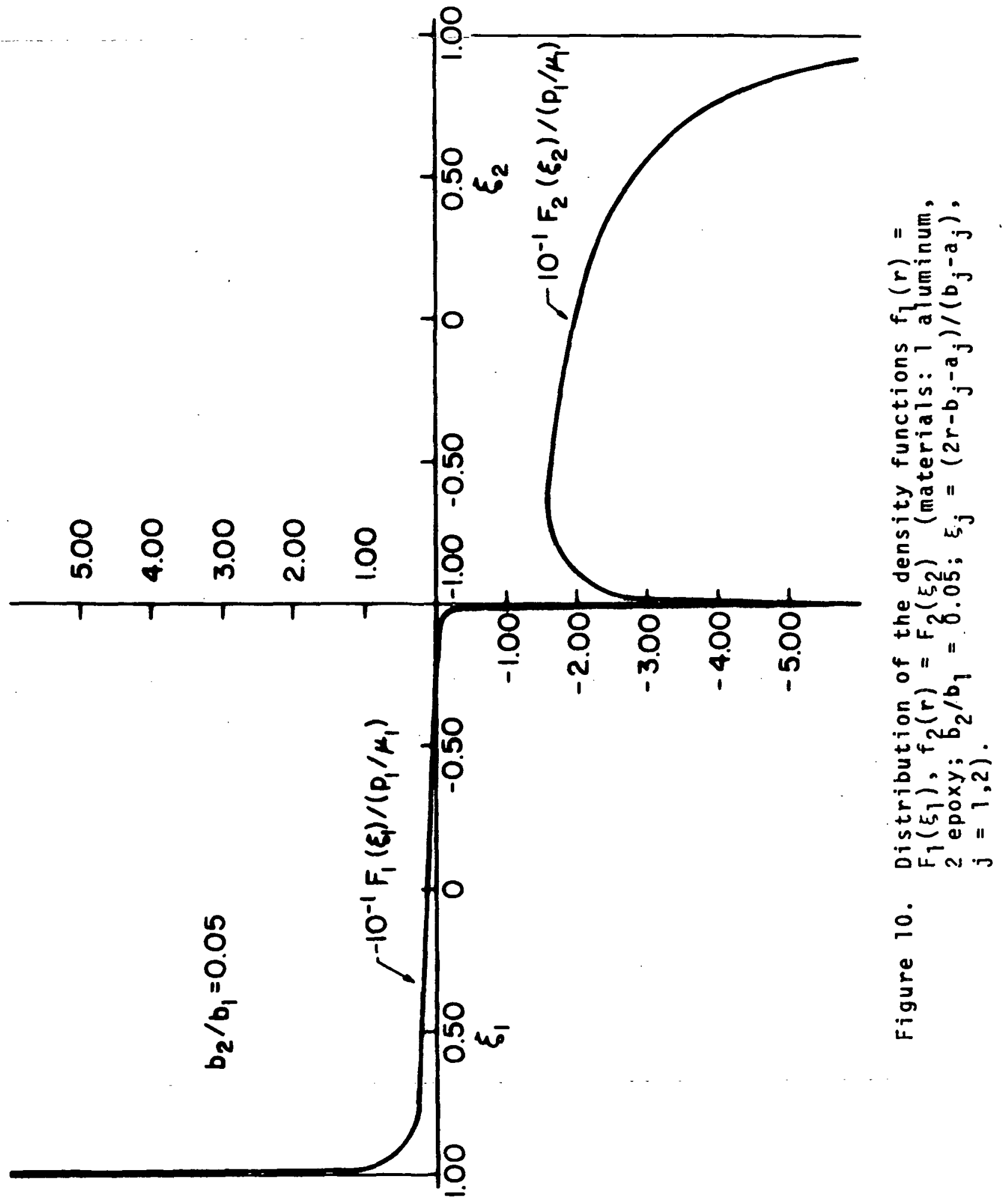


The results shown in Figures 11,12 , and 13 correspond to the examples given in the Tables 3,4 , and 5 , respectively. In Figures 11 and 13 the length unit for displacements is $b_{1}$ and in Figure 12 it is $\ell=\left(b_{1}+b_{2}\right) / 2$. A careful examination of the crack surface displacements in the adjacent materials around $r=0$ would indicate that the crack opening in the medium with the higher modulus is greater than that with the lower modulus, both having an infinite slope.

The results given in this paper may be used in the analysis of fracture propagation in the composite medium by using any of the standard fracture criteria if the crack extension takes place at the singular point $r=b_{1}$ or $r=b_{2}$ where the singularity is of the form $\left(r-b_{j}\right)^{-1 / 2}$. However, in studying a possible fracture initiation at $r=0$ (where the singularity is of the form $r^{-\alpha}$ ), i.e., initiation of a debonding crack along the interface or a cleavage crack in one of the adjacent materials, a criterion similar to that outlined in [8] may have to be used. 
Page Intentionally Left Blank 


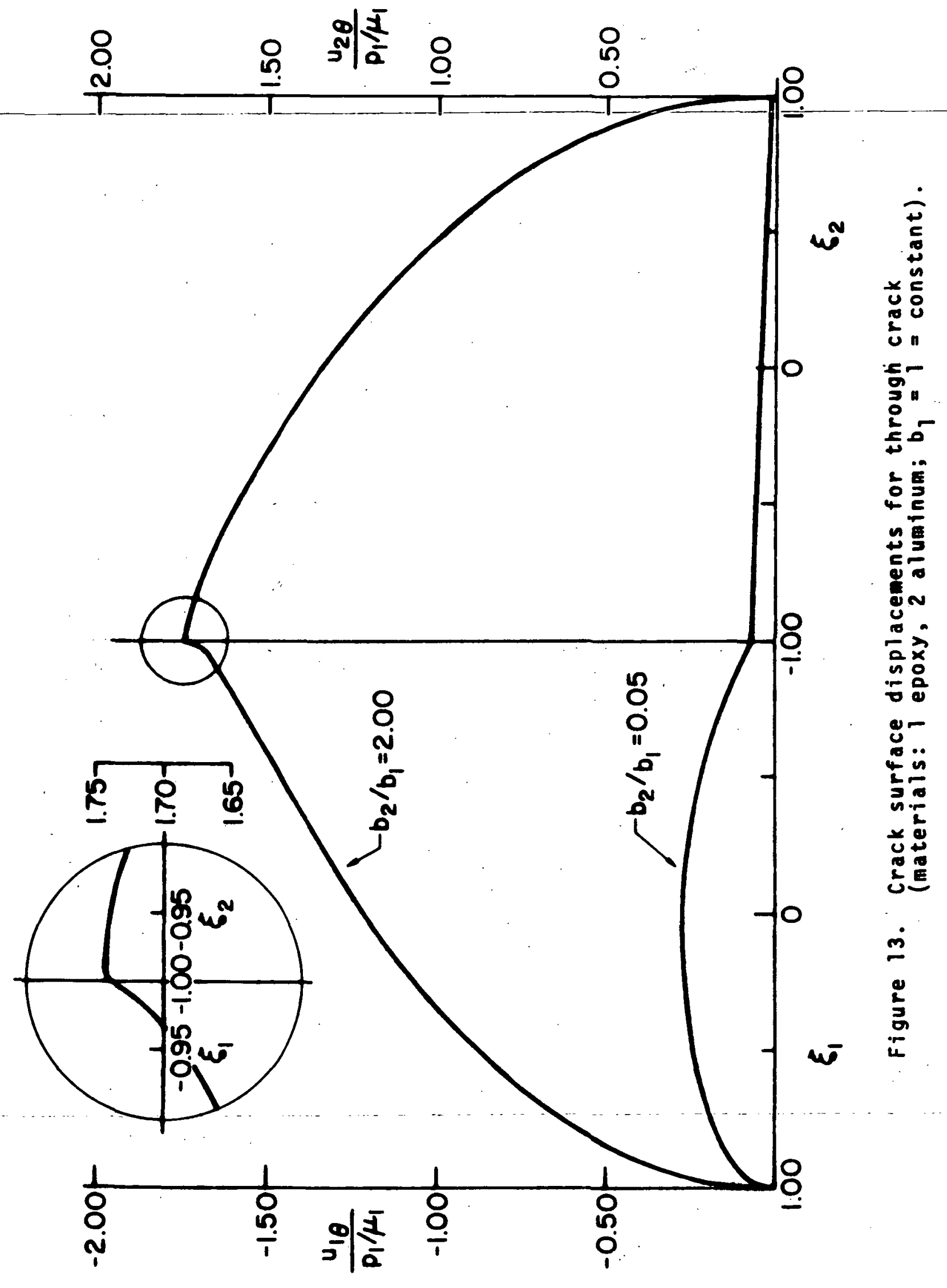




\section{REFERENCES}

1. F. Erdogan and G. D. Gupta, "Stress Analysis of MultiLayered Composites with a Flaw", Int. J. Solids, Structures, Vo1. 7, p. 39 (1971).

2. F. Erdogan and G. D. Gupta, "Layered Composites with an Interface Flaw", Int. J. Solids, Structures, Vol. 7 , p. 1089 (1971).

3. K. Arin and F. Erdogan, "Penny-Shaped Crack in an Elastic Layer Bonded to Dissimilar Half Spaces", Int. J. Engng. Sci., Vol. 9, p. 213 (1971).

4. F. Erdogan and K. Arin, "Penny-Shaped Interface Crack Between an Elastic Layer and a Half Space", Int. J. Engng. Sci.Vol. 10, p. 115 (1972).

5. F. Erdogan and T. Ozbek, "Stresses in Fiber-Reinforced Composites with Imperfect Bonding", J. Appl. Mech., Vol. 36, p. 865, Trans. ASME, Series E (1969).

6. F. Erdogan and G. D. Gupta, "Stresses Near a Flat Inclusion in Bonded Dissimilar Materials", Int. J. Solids, Structures, Vol. 8, p. 533 (1972).

7. F. Erdogan, "Fracture Problems in Composite Materials", J. Engineering Fracture Mechanics (to appear, 1972).

8. T. S. Cook and F. Erdogan, "Stresses in Bonded Materials with a Crack Perpendicular to the Interface", Int. J. Engng. Sci. (to appear, 1972).

9. F. Erdogan and G. D. Gupta, "On the Numerical Solution of Singular Integral Equations", Quarterly of Applied Mathematics, p. 525 (1972).

10. N. I. Muskhelishvili, Singular Integral Equations, P. Noordhoff, Groningen, The Netherlands (1953).

11. D. B. Bogy, "Edge-Bonded Dissimilar Orthogonal Elastic Wedges Under Normal and Shear Loading", J. Appl. Mech., Vor. 35, Trans. ASME, Series E, p. 460 (1968).

12. V. L. Hein and F. Erdogan, "Stress Singularities in a Two-Material Wedge", Int. J. Fracture Mechanics, Vol. 7, p. 317 (1971).

13. H. F. Bueckner, "On a Class of Singular Integral Equations", J. Math. Analysis and Applications, Vol. 14, p. $392(1966)$.

14. G. T. Bierman, "A Particular Class of Singular Integral Equations", SIAM J. App 1. Math., Vo1. 20, p. 99 (1971).

15. F. Erdogan, "Complex Function Technique", in Treatise on Continuum Physics, A. C. Eringen, Editor, Vol. II, Part III, Chapter 2, (1972). 
"The aeronautical and space activities of the United States shall be conducted so as to contribute ... to the expansion of buman knowledge of phenomena in the atmosphere and space. The Administration shall provide for the widest practicable and appropriate dissemination of information concerning its activities and the results thereof."

-National aeronautics and SpaCe ACt of 1958

\section{NASA SCIENTIFIC AND TECHNICAL PUBLICATIONS}

TECHNICAL REPORTS: Scientific and technical information considered important, complete, and a lasting contribution to existing knowledge.

TECHNICAL NOTES: Information less broad in scope but nevertheless of importance as a contribution to existing knowledge.

TECHNICAL MEMORANDUMS:

Information receiving limited distribution because of preliminary data, security classification, or other reasons. Also includes conference proceedings with either limited or unlimited distribution.

CONTRACTOR REPORTS: Scientific and technical information generated under a NASA contract or grant and considered an important contribution to existing knowledge.
TECHNICAL TRANSLATIONS: Information published in a foreign language considered to merit NASA distribution in English.

SPECIAL PUBLICATIONS: Information derived from or of value to NASA activities. Publications include final reports of major projects, monographs, data compilations, handbooks, sourcebooks, and special bibliographies.

\section{TECHNOLOGY UTILIZATION}

PUBLICATIONS: Information on technology used by NASA that may be of particular interest in commercial and other non-aerospace applications. Publications include Tech Briefs, Technology Utilization Reports and Technology Surveys.

Details on the availability of these publications may be obtained from:

SCIENTIFIC AND TECHNICAL INFORMATION OFFICE 